\title{
Toward Real-Time Monitoring and Control of Single Nanoparticle Properties with a Microbubble Resonator Spectrometer
}

\author{
Levi Hogan, Erik Horak, Jonathan Ward, Kassandra Knapper, Síle Nic Chormaic, Randall Goldsmith
}

Submitted date: 20/06/2019 - Posted date: 20/06/2019

Licence: CC BY-NC-ND 4.0

Citation information: Hogan, Levi; Horak, Erik; Ward, Jonathan; Knapper, Kassandra; Nic Chormaic, Síle; Goldsmith, Randall (2019): Toward Real-Time Monitoring and Control of Single Nanoparticle Properties with a Microbubble Resonator Spectrometer. ChemRxiv. Preprint.

Optical microresonators have widespread application at the frontiers of nanophotonic technology, driven by their ability to confine light to the nanoscale and enhance light-matter interactions. Microresonators form the heart of a new method for single-particle photothermal absorption spectroscopy, whereby the microresonators act as microscale thermometers to detect the heat dissipated by optically pumped, non-luminescent nanoscopic targets. However, translation of this technology to chemically dynamic systems requires a platform that is mechanically stable, solution compatible, and visibly transparent. We report microbubble absorption spectrometers as a new and versatile platform that meets these requirements. Microbubbles integrate a two-port microfluidic device within a Whispering Gallery Mode (WGM) microresonator, allowing for the facile exchange of chemical reagents within the resonator's interior while maintaining a solution-free environment on its exterior. We first leverage these qualities to investigate the photo-activated etching of single gold nanorods by ferric chloride, providing a new method for rapid acquisition of spatial and morphological information about nanoparticles as they undergo chemical reactions. We then demonstrate the ability to control nanorod orientation within a microbubble through optically exerted torque, a new route toward the construction of hybrid photonic-plasmonic systems. Critically, the reported platform advances microresonator spectrometer technology by permitting room-temperature, aqueous experimental conditions, opening a regime of time-resolved single-particle experiments on non-emissive, nanoscale analytes engaged in catalytically and biologically relevant chemical dynamics.

File list (2)

Microbubble Main Text ChemRxiv v2.pdf (1.98 MiB)

view on ChemRxiv • download file 


\title{
Toward Real-Time Monitoring and Control of Single Nanoparticle Properties with a Microbubble Resonator Spectrometer
}

\author{
Levi T. Hogan ${ }^{\dagger, 1}$, Erik H. Horak ${ }^{\dagger, 1}$, Jonathan M. Ward ${ }^{2}$, Kassandra A. Knapper ${ }^{1}$, Síle Nic \\ Chormaic $^{2}$, Randall H. Goldsmith*,1
}

\begin{abstract}
Optical microresonators have widespread application at the frontiers of nanophotonic technology, driven by their ability to confine light to the nanoscale and enhance light-matter interactions. Microresonators form the heart of a new method for single-particle photothermal absorption spectroscopy, whereby the microresonators act as microscale thermometers to detect the heat dissipated by optically pumped, non-luminescent nanoscopic targets. However, translation

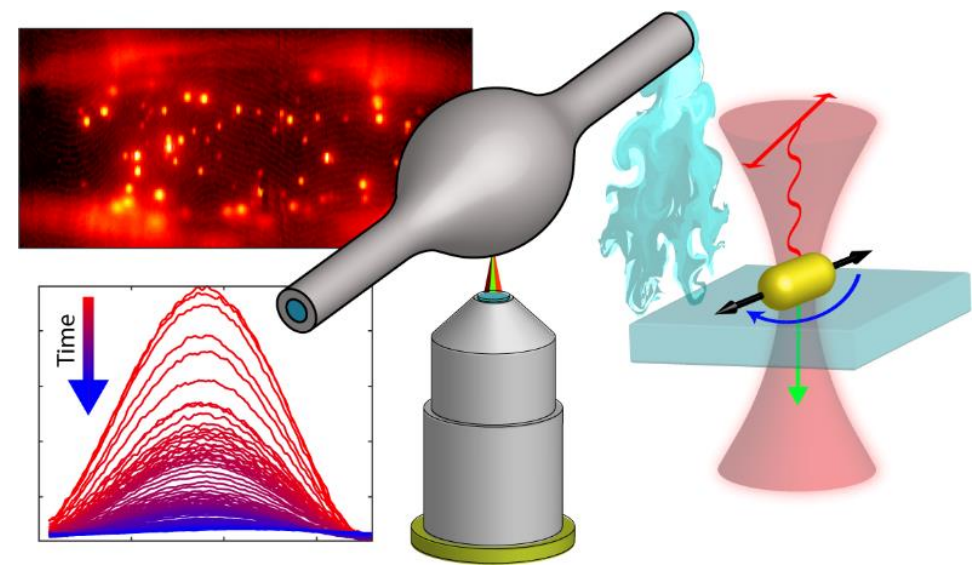
of this technology to chemically dynamic systems requires a platform that is mechanically stable, solution compatible, and visibly transparent. We report microbubble absorption spectrometers as a new and versatile platform that meets these requirements. Microbubbles integrate a two-port microfluidic device within a Whispering Gallery Mode (WGM) microresonator, allowing for the facile exchange of chemical reagents within the resonator's interior while maintaining a solution-free environment on its exterior. We first leverage these qualities to investigate the photo-activated etching of single gold nanorods by ferric chloride, providing a new method for rapid acquisition of spatial and morphological information about nanoparticles as they undergo chemical reactions. We then demonstrate the ability to control nanorod orientation within a microbubble through optically exerted torque, a new route toward the construction of hybrid photonicplasmonic systems. Critically, the reported platform advances microresonator spectrometer technology by permitting room-temperature, aqueous experimental conditions, opening a regime of time-resolved singleparticle experiments on non-emissive, nanoscale analytes engaged in catalytically and biologically relevant chemical dynamics.
\end{abstract}

${ }^{1}$ Department of Chemistry, University of Wisconsin-Madison, Madison, Wisconsin 53706, United States

${ }^{2}$ Light-Matter Interactions Unit, Okinawa Institute of Science and Technology Graduate University, Onna, Okinawa 904-0495, Japan

$\dagger$ These authors contributed equally to this work.

*rhg@chem.wisc.edu 


\section{Toward Real-Time Monitoring and Control of Single Nanoparticle Properties with a Microbubble Resonator Spectrometer}

Levi T. Hogan ${ }^{\dagger}$, Erik H. Horak ${ }^{\dagger}$ Jonathan M. Ward, Kassandra A. Knapper, Síle Nic Chormaic, Randall H. Goldsmith*

Optical microresonators, devices that confine light to microscopic volumes, have found widespread application within chemistry, biology, physics, and engineering..$^{1-5}$ A broad class of optical microresonators, whispering-gallery mode (WGM) resonators, have exhibited superb sensitivity including the detection of single nanoparticles, ${ }^{6,7}$ single molecules ${ }^{8-11}$ and even single metal ions. ${ }^{12}$ However, the ability to perform spectroscopy on adsorbed objects would not only open a path toward label-free chemical identification, but also allow the interrogation of single object properties, free from the static and dynamic blurring of typical ensemble measurements. To this end, we recently employed microtoroid resonators as single-particle absorption spectrometers, whereby the heat dissipated by optically pumped nano-objects such as gold nanorods (AuNRs), ${ }^{13-16}$ carbon nanotubes, ${ }^{17}$ or conductive polymers ${ }^{18}$ is detected via small shifts in the WGM resonance condition. However, to harness the sensitivity of this method for chemically dynamic systems, a platform easily compatible with solution-phase measurements is necessary. Here, we report such a platform, the microbubble resonator, and use it to study the photo-activated chemical etching and reorientation of single AuNRs.

AuNRs ${ }^{19}$ have important chemical and biological ${ }^{20}$ applications such as bioimaging, ${ }^{21}$ treatment of cancer $^{22,}{ }^{23}$ and infection, ${ }^{24}$ label-free biosensing ${ }^{25}$ down to single molecules, ${ }^{26}$ surface-enhanced Raman spectroscopy, ${ }^{27,}{ }^{28}$ fluorescence enhancement, ${ }^{29}$ drug delivery, ${ }^{30}$ and light harvesting to drive catalytic reactions. ${ }^{31}$ These applications heavily rely on tuning the morphology-dependent optical features of AuNRs, necessitating precise tailoring of their dimensions. This result can be achieved during AuNR fabrication, where seed-mediated synthesis ${ }^{32}$ can often tame the polydispersity that typically plagues samples. However, in many cases polydisperse AuNR samples are still common and post-synthetic modifications offer an attractive route to achieve a desired morphology. Furthermore, significant particle-to-particle variations of key optical properties of AuNRs both on surfaces ${ }^{33}$ and in solution ${ }^{34}$ highlight the heterogeneity within a population of nanoparticles, underscoring the need for single-particle inspection, including during nanoparticle synthesis and modification. A variety of optical methods exist for probing non-luminescent single nanoparticles and molecules via photothermal, ${ }^{35-38}$ scattering, ${ }^{39-41}$ and other techniques. ${ }^{42-45}$ Observation of the chemical etching of single AuNRs has recently been accomplished with one-photon luminescence, ${ }^{46,} 47$ dark-field scattering, ${ }^{48-53}$ and liquid transmission electron microscopy (TEM). ${ }^{54}$ However, a highly sensitive absorption technique for monitoring such chemical dynamics is needed to compliment these methods, and would be extremely valuable for accessing targets that are not luminescent or are too small for scattering experiments. WGM resonators are perfectly poised to fill this gap in methodology.

Various WGM microresonator geometries have been employed for sensing in solution, including microspheres, ${ }^{9-11}$ microrings, ${ }^{5,}{ }^{55}$ microtoroids,,${ }^{6,56}$ microbubbles, ${ }^{57-59}$ microdroplets, ${ }^{60}$ microtubes, ${ }^{61}$ and microbottles. ${ }^{62,63}$ In particular, the variations and capabilities of hollow microresonators for sensing have been reviewed in detail elsewhere. ${ }^{64}$ To adapt a microresonator for in-solution, visible-wavelength photothermal spectroscopy, three requirements must be met: (i) high sensitivity for interrogating nanoscopic analytes, (ii) resonator transparency at visible wavelengths to mitigate photothermal background, and (iii) robust performance in solution. Employing silica-on-silicon $\left(\mathrm{SiO}_{2}-\mathrm{Si}\right)$ microtoroids for photothermal spectroscopy, one can resolve attometer shifts of the WGM resonant wavelength from thermal fluxes of target nano-objects. ${ }^{13}$ High backgrounds in $\mathrm{SiO}_{2}$-Si toroids can be mitigated with all-glass microtoroids, which can be used for visible spectroscopy. ${ }^{15}, 16$ However, immersing a WGM microresonator in water mandates the use of larger microresonators to avoid bending losses, ${ }^{65}$ with consequent lower 
photothermal sensitivity. Furthermore, although tapers ${ }^{66}$ and prisms ${ }^{9}$ can be optically coupled to WGM microresonators in water, immersion of such couplers in solution may reduce mechanical stability and also result in fouling, particularly as more caustic reagents are employed for chemical studies. Therefore, an alternative platform is preferable for in-solution experiments. The microbubble WGM resonator, Figure 1B, which possesses a hollow, solution-accessible interior, while maintaining an air-glass exterior interface, meets the requirements for in-solution, visible spectroscopy of nanoscopic analytes.

Microbubbles are fabricated from glass capillaries, resulting in low background signals at visible wavelengths, tunable fabrication, and two-port connectivity through which it is easy to flow reagents. Compared to a solid resonator immersed in solution, a microbubble maintains an air-glass interface on its exterior, enabling a higher refractive index contrast and allowing for smaller diameter resonators before bending losses occur. Additionally, the tapered optical fiber used for coupling light into the resonator can approach in air, reducing noise from the instability of coupling in-solution and eliminating solution contamination of the taper. Furthermore, the unique, thin-walled structure of the microbubble allows for high-order optical modes that exist almost entirely within the liquid-core of the resonator, a situation termed the "quasi-droplet regime". Operating in the quasi-droplet regime, microbubbles have proven exceptional sensors, most recently for detecting polystyrene nanoparticles in aqueous solution with a sensitivity 280 times larger than similar experiments using microsphere resonators. ${ }^{57,58}$ Together, these factors make microbubble resonators ideal for time-resolved spectroscopy of single-particle chemical reaction dynamics when exposed to solution. In this paper, we introduce microbubble absorption spectrometers for probing and controlling the chemical and rotational dynamics associated with the photo-activated chemical etching of AuNRs. This platform holds promise for elucidating mechanistic insights into nanoparticle reactions with a method orthogonal to existing techniques that rely on scattering or luminescence, and is an attractive candidate for future single-particle and single-molecule studies.

\section{Experimental Design:}

WGM resonators operate via total internal reflection, wherein light propagates around a closed geometric loop, resulting in resonance conditions where only specific wavelengths propagate constructively. WGM resonances are interrogated by the "probe beam", provided by a continuous wave $(\mathrm{CW})$, narrow-linewidth, tunable laser coupled through a tapered optical fiber ${ }^{67}$ (Figure 1A). Transmitted light through the tapered fiber is collected and the probe beam is actively locked to a resonance by a PoundDrever-Hall locking system. ${ }^{6,13,68-70}$ The hollow core of the microbubble is filled with the desired reagents by attaching the microbubble capillary to a syringe pump. Two microbubbles are pictured in Figure 1B, highlighting the tunability of geometric parameters, and consequent versatility on optofluidic properties.

A second beam, the "pump beam", is focused onto the microbubble surface to excite analytes. CW diode lasers at $532 \mathrm{~nm}, 635 \mathrm{~nm}$, and $785 \mathrm{~nm}$ are coaligned using dichroic mirrors, permitting interrogation at different wavelengths. The linearly polarized pump beam is amplitude modulated at $433 \mathrm{~Hz}$ using an optical chopper, encoding the photothermal signal at this frequency and allowing for use of lock-in amplification to drastically lower the experimental noise floor. ${ }^{13}$ Two galvanometer mirrors steer the pump beam through a relay lens system to a 40x objective with piezo-controlled focus, providing spatial control of the pump beam on the microbubble resonator. This spatial control is leveraged to photothermally map the interior surface of microbubble resonators, at low resolution for an entire resonator, and high resolution for single diffraction-limited objects. Figure 1C shows two photothermal maps of a microbubble resonator at different objective foci, with the out-of-focus map indicating the curvature of the microbubble from the varied PSFs across the map. The polarization angle of the linearly polarized pump beam is rapidly scanned using a voltage-controlled liquid crystal, which is sandwiched between a polarizer and a quarter-waveplate (see Methods). This combination of wavelength, spatial, and polarization control permits thorough 
characterization of individual analytes bound to the resonator, realized at exquisite sensitivity due to the double-modulation scheme.

A)

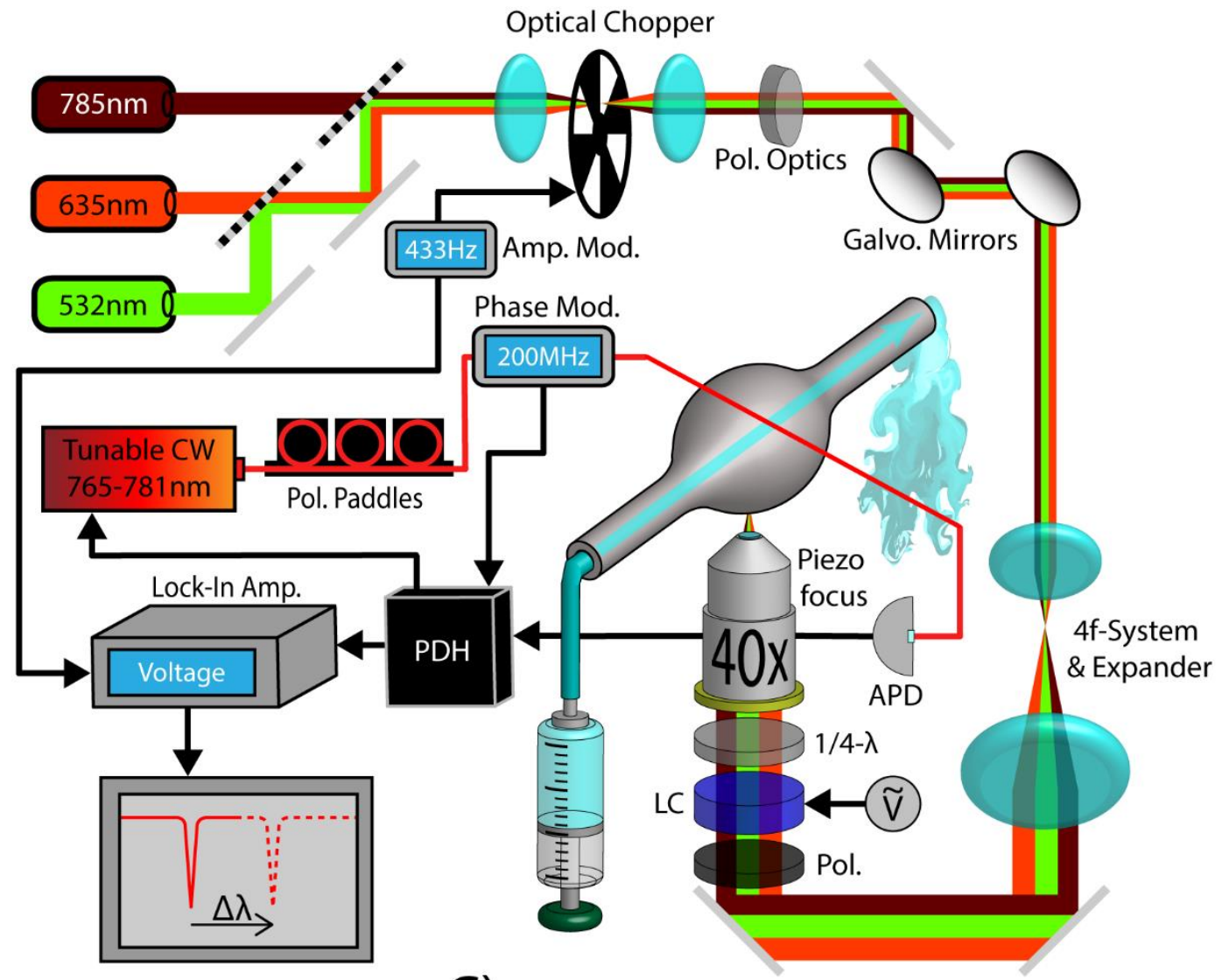

B)

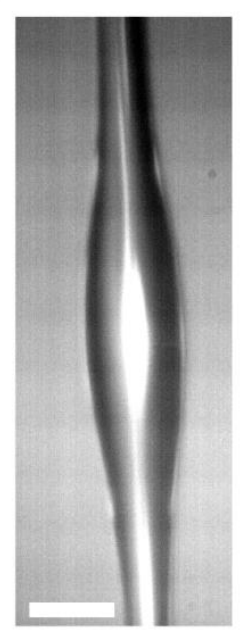

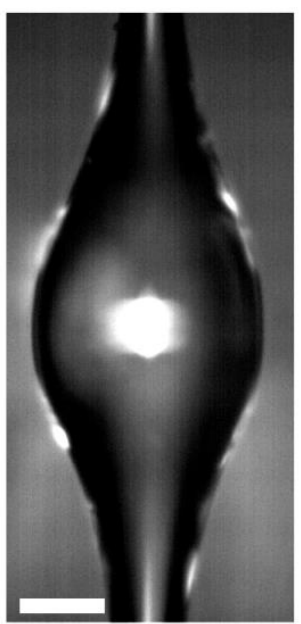

C)
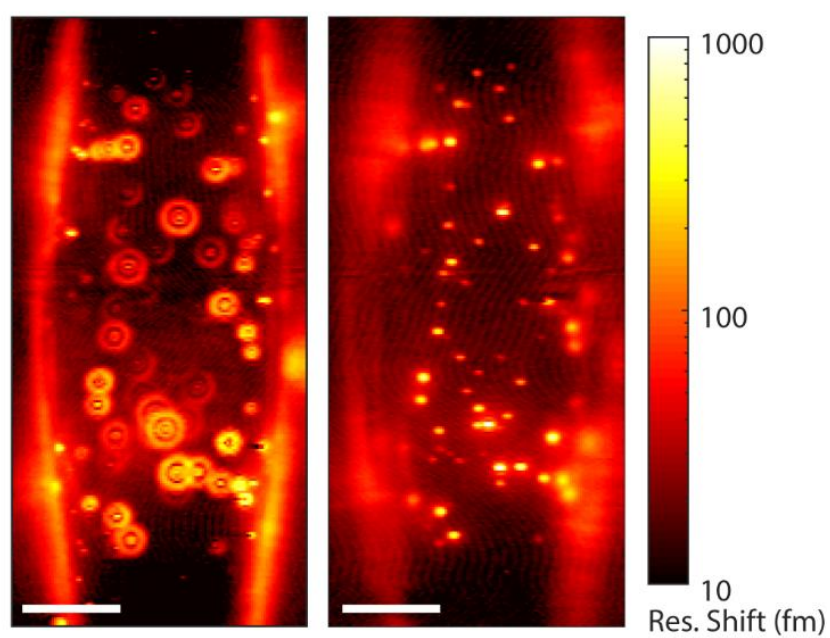

Figure 1. Microbubble Absorption Spectroscopy. (A) Cartoon of instrumentation. PDH=Pound Drever Hall. LC=Liquid Crystal. APD=Avalanche Photodiode. (B) Optical micrographs of two microbubble resonators with different geometries. Scale bars 20 um. C) Photothermal maps of a microbubble resonator similar in geometry to the left microbubble in Figure 1B, both out-of-focus (left), and in-focus (right). Scale bars $20 \mathrm{um}$. 


\section{Operation of Single-Particle Microresonator Spectrometers:}

Microresonator sensing schemes generally rely on the reactive mechanism, ${ }^{10}$ whereby binding of an analyte imparts a small refractive index change, shifting the resonance wavelength. Instead, microresonator-based photothermal spectroscopy relies on a resonance shift resulting from the heat plume generated by optically pumping a non-emissive object bound to the resonator surface. ${ }^{17}$ The temperature rise accompanying this heat plume alters the resonator's refractive index according to its thermo-optic coefficient $(d n / d T)$, changing the WGM optical path length and shifting the resonance condition. The ability to detect this resonance shift is related to the figure of merit $\mathrm{Q} / \mathrm{V}$, the ratio of the resonator's quality factor (Q) and mode volume (V). A resonator with minimized absorption, bending, and scattering losses allows photons to repeatedly circulate the resonator, resulting in a high $\mathrm{Q}$, narrow linewidth resonance. This narrow linewidth increases the visibility of minute resonance shifts. A smaller resonator with consequent tighter confinement of light produces a smaller $\mathrm{V}$, increasing the overlap between the thermal plume of the analyte and the optical mode. This increased overlap contributes a larger effective refractive index change and thus a larger resonance shift. ${ }^{17}$ To properly examine the microbubble photothermal response we employed finiteelement simulations (COMSOL) of both the optical modes and the thermal properties of the microresonator. Simulated optical modes for a particular microbubble geometry are shown in Figure 2A. Varying mode numbers, defined in the traditional spherical geometric indices (polar, azimuthal, and radial, Supporting Information), clearly show the complicated mode structure inherent in the microbubble resonators. This complex mode structure gives rise to several important experimental considerations.

First, the efficient excitation of high-order modes leads to incredibly congested mode spectra. An illustrative 180 pm window of a water-filled microbubble's resonance landscape is shown in Figure 2B. This high mode density stems from the highly prolate resonator geometry lifting the polar mode degeneracy relative to an ideal spherical resonator, ${ }^{71}$ leading to varying effective resonator sizes for modes, as well as differing free-spectral ranges. ${ }^{72}$ The differing free-spectral ranges cause spectral overlap of modes of different azimuthal mode order ${ }^{73}$ an effect that is compounded by the disparate dielectric environments experienced by different-order radial modes, which have different fractions of the electric field contained in glass, water, and air. Second, the burrowing of higher-order radial modes into the water-filled interior not only changes the effective refractive index of the mode but also yields tremendous variations in $d n / d T$. This varied $d n / d T$, which can even switch signs, produces very different thermal responsivities for modes. The combined congested mode spectrum and differential shifting from $d n / d T$ variation amplifies experimental challenges, as photothermal heating or ambient temperature drifting can cause modes to shift through each other. Therefore, modes that are both thermally responsive and spectrally isolable are desirable.

Choosing a high thermal responsivity mode requires delving into the expected thermal response with finite element simulations. As described above, the radial mode order drastically alters the effective $d n / d T$, as glass has a small positive $d n / d T$ of $9 \times 10^{-6} \mathrm{~K}^{-1}$ and water a large negative $d n / d T$ of $-91 \times 10^{-6} \mathrm{~K}^{-1}$. Thus, while glass-contained modes in a water-filled resonator offer $\mathrm{Q}$ values over $10^{6}$ and show small positive resonance shifts upon heating, higher-order, water-contained modes offer Q values of mid-10 5 and show large negative resonance shifts captured both experimentally (Supporting Information) and in our simulations in Figure 2C. The "shark fin" shape results from the pump beam amplitude modulation. Interestingly, this modulation rides atop a rising baseline magnitude as heat builds over many modulation cycles (left panel) before thermal equilibrium is reached (right panel). This baseline stems from the lack of an effective, proximal heat sink in microbubbles with equilibration reached only after sufficient heat dissipation to the air, yielding a baseline shift about ten times larger than the modulating shift, both experimentally and theoretically (Supporting Information). 
Finding high-order water-contained radial modes to leverage their larger thermal response requires careful consideration of the coupling geometry. Specifically, the tapered fiber diameter ${ }^{72}$ greatly impacts mode selectivity through phase matching conditions and evanescent field overlap. By translating along the length of the tapered fiber this diameter was tuned until these water-contained modes were suitably excited. Then a thermally sensitive and spectrally isolated mode was selected by wavelength scanning. Importantly, the precise identity of this mode was not discerned, precluding direct relation of a resonance shift with an absolute absorption cross-section as in our previous experiments. ${ }^{13},{ }^{17}$ Identification is possible, ${ }^{74-76}$ particularly when implementing procedures to simplify the mode structure, ${ }^{73,77}$ but difficult in practice and was not pursued here.

Importantly, this lack of mode identification precludes the selection of the maximally thermally responsive mode. While the optimal mode is required for ideal sensor response, use of a less responsive resonance was sufficient for examination of AuNRs. The limit-of-detection of our system was investigated by optically pumping a single AuNR inside a resonator. The photothermal signal, averaged for $30 \mathrm{~s}$ with a

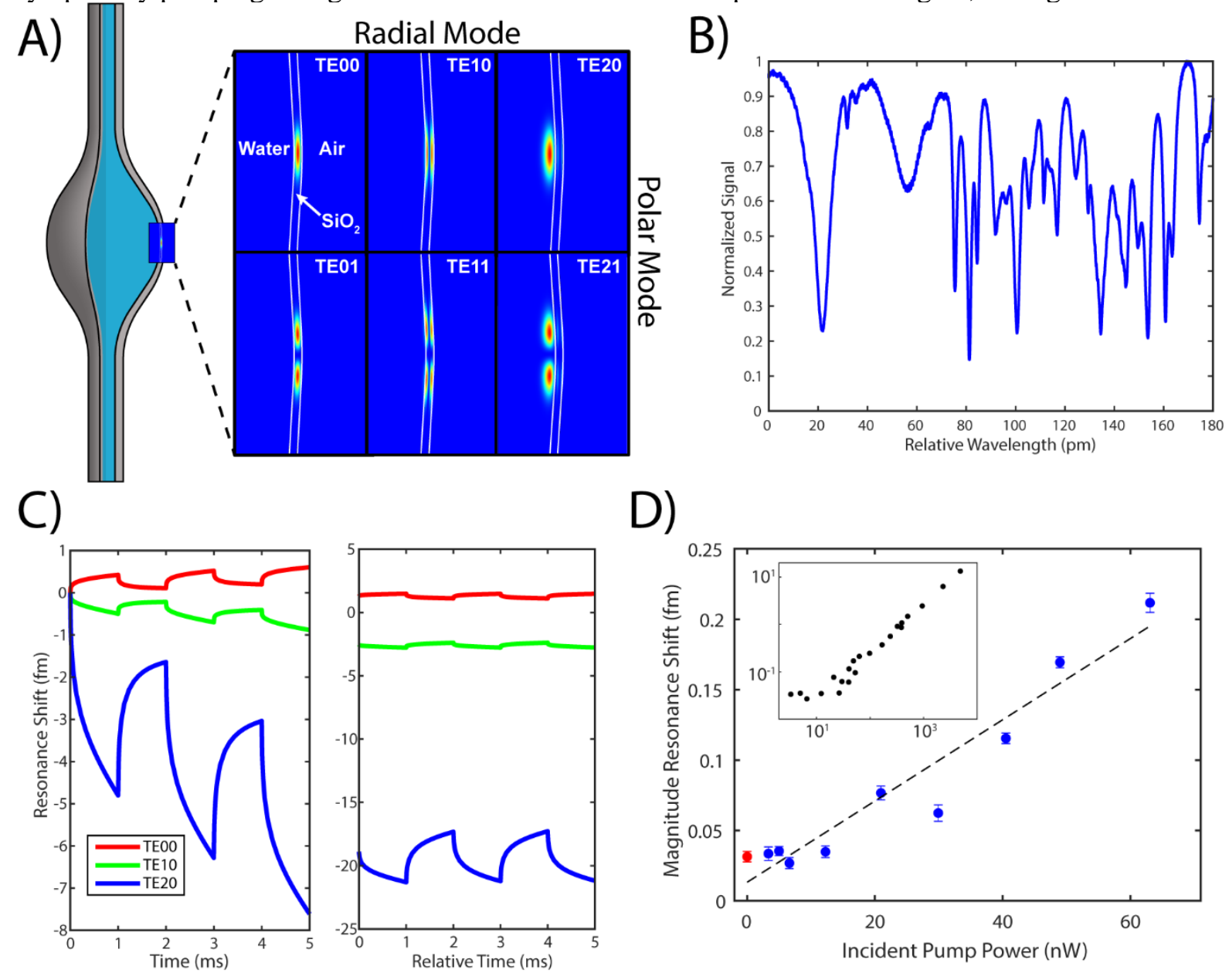

Figure 2. Optical resonances in microbubble resonators. (A) Simulated electric field distributions at $780 \mathrm{~nm}$ for first, second, and third order radial modes, for both first and second order polar modes. All modes shown are Transverse Electric (TE). White curves are added to clearly indicate the position of the microbubble walls. (B) A 180 pm span of the mode spectrum of a microbubble resonator. (C) Left: The signal at the beginning of analyte pumping. Right: Signal once the resonator has reached a thermal equilibrium with its surroundings (theoretical). (D) Resonance shift from pumping a single gold nanorod with the $635 \mathrm{~nm}$ beam at decreasing powers (blue points). The red point indicates the signal for pump beam off. The inset is a zoom-out, showing signal linearity over orders of magnitude in pumping power. Further details in main text. Error bars are standard deviation of the mean. 
time constant of $1 \mathrm{~s}$ (Figure 2D), was monitored at decreasing powers until it was indistinguishable from the signal obtained with the pump beam blocked. As the inset in Figure 2D shows, the signal remains linear over multiple orders of magnitude, flattening out at low powers as the noise floor is reached. The detection limit for this platform is in the low tens of attometers of wavelength shift (a comparison with microtoroids is made in the Supporting Information). For context, the typical photothermal response of a single AuNR at our pump fluxes is in the range of 10-100 fm, easily resolvable by many orders of magnitude. Additionally, this detection limit surpasses the expected femtometer photothermal shift for measuring a single chromophore. ${ }^{17}$ As a first step towards monitoring reaction dynamics of molecules, we show below that microbubbles are well-suited for probing the chemical and spatial dynamics of single AuNRs.

\section{Probing photophysical features of single AuNRs:}

AuNRs exhibit optical features known as localized surface plasmon resonances (LSPRs), which result from light exciting collective oscillations of conduction band electrons. Two orthogonal LSPRs exist in AuNRs: the longitudinal plasmon band (LPB) and the transverse plasmon band (TPB), oriented parallel and perpendicular, respectively, to the long axis of the rod (Figure 3A). The LPB is at the longer wavelength in the bulk extinction spectra of the AuNRs used in this report $(80 \times 40 \mathrm{~nm})$, Figure 3B. These spectral features are probed with the microbubble platform detailed in Figure 1A at the single AuNR level, at specific pump beam wavelengths (solid vertical lines Figure 3B). The LPB central wavelengths will likely be red-shifted compared to the bulk due to interaction with the glass surface. ${ }^{39,78,79}$

After depositing AuNRs inside of a microbubble resonator (Methods), the resonator is photothermally mapped to find objects. To confirm successful deposition of single AuNRs, photothermal maps are acquired with the pump beam, linearly polarized from 0 to 180 (Figure 3C) at three different pump wavelengths. These maps are fit to extract an intensity at each polarization (Methods), shown as data points in Figure 3D. These polarization dependent intensities are fit (dotted lines) to give a depth-ofmodulation, M (Methods). The $635 \mathrm{~nm}$ and $785 \mathrm{~nm}$ traces, which probe the LPB, have a value of M close to unity for a single AuNR. A criterion of $\mathrm{M} \geq 0.98$ was used for classifying an object as a single AuNR. Small well-ordered aggregates could also exhibit high $\mathrm{M}$ values, but these are unlikely due to the presence of CTAB during deposition. Alternatively, a much faster method of probing AuNR orientation is to rapidly rotate the pump beam's linear polarization while centered on an object, and fit the results to extract $\mathrm{M}$. Although this method (Figure 3E) lacks background subtraction, it allows for hundreds of data points to be collected in a few seconds, resulting in quick determination of AuNR orientation and relative absorption cross-section at the pumping wavelength during reactions.

For both of the above polarization methods, the traces for the $635 \mathrm{~nm}$ and $785 \mathrm{~nm}$ pump beams align in peak angle (Figure 3D and Figure 3E) because both of these wavelengths excite the LPB. In contrast, the $532 \mathrm{~nm}$ trace has a peak angle orthogonal to the other two traces because this wavelength excites the TPB at a pump polarization orthogonal to the LPB excitation. In addition, the $532 \mathrm{~nm}$ trace does not go to zero, because at that wavelength the pump beam is not only pumping the polarization-dependent $\mathrm{TPB}$, but also the interband transitions of gold, which are independent of pump beam polarization. Notably, the use of multiple wavelengths means that AuNRs can not only be localized, but also studied spectroscopically. Herein, these capabilities are employed to study the etching of AuNRs in real-time.

\section{Selecting an etchant:}

The photophysical properties of AuNRs are well understood as a function of geometry ${ }^{80}$ and postsynthetic modifications are extremely useful for exerting control over these properties. Since 2002, when Jana and coworkers observed anisotropic etching of gold spheroids in both cyanide and persulfate solutions, ${ }^{81}$ at least 20 other reagents have been reported to etch or accelerate the etching of AuNRs, often with spatial selectivity (see Supporting Information). Such reports include assays for facile detection of 
A)

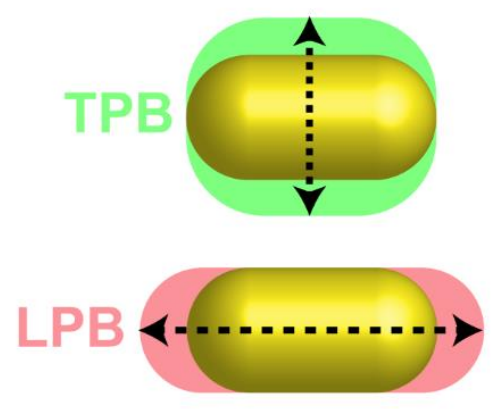

C)
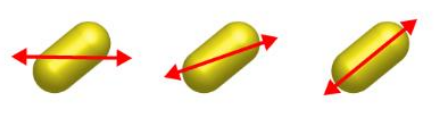

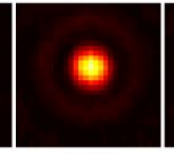

$20^{\circ}$
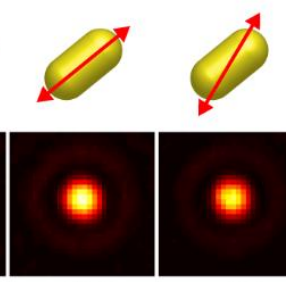

$40^{\circ}$

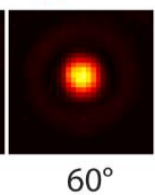

D)
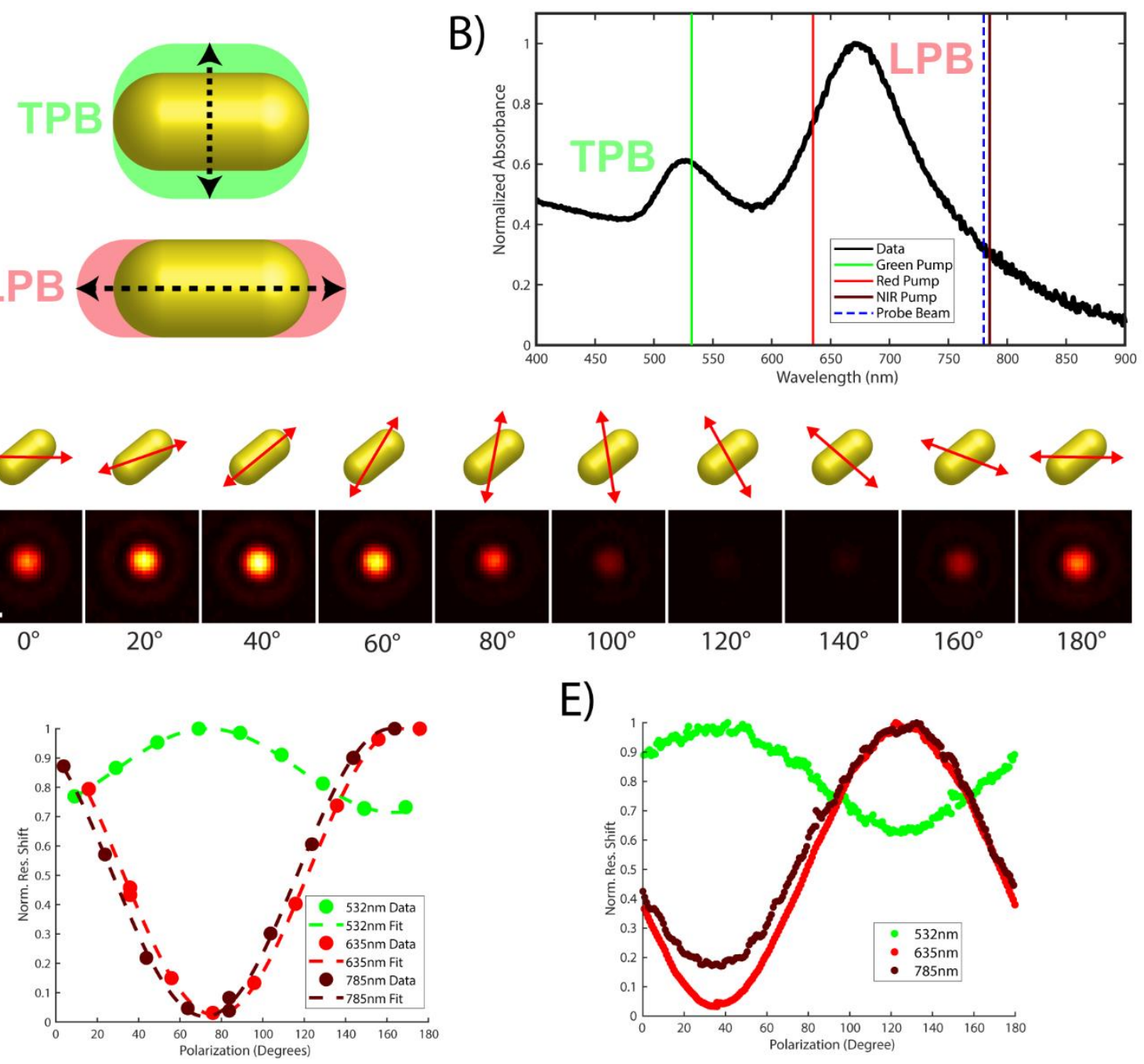

E)

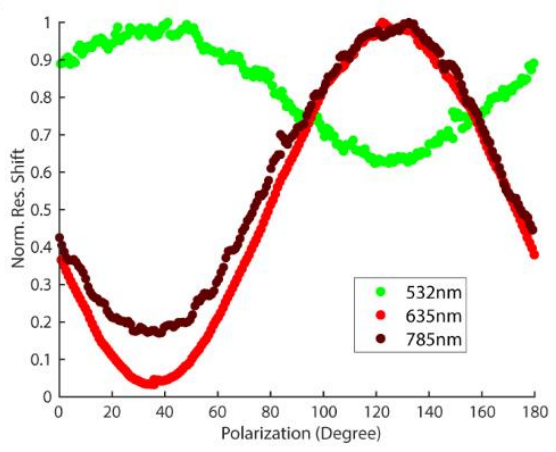

Figure 3. Probing photophysical features of single AuNRs. (A) Cartoon illustrating the photophysical features of a AuNR. LPB=Longitudinal Plasmon Band. TPB=Transverse Plasmon Band. (B) Bulk absorption spectrum of AuNRs, with the various laser beams in our experiment indicated by vertical lines. LPB and TPB indicated. (C) Example photothermal maps of a nanorod as pump polarization is varied in increments of 20 degrees, as shown by the red arrow in the cartoon above the photothermal maps. Scale bar $1 \mu \mathrm{m}$. (D) Polarization fits for three different pump beams acquired using photothermal mapping. (E) Polarization traces for three different pump beams, acquired by recording photothermal signal as the linear pump polarization is quickly rotated 180 degrees ( 10 seconds).

analytes at ultra-low concentrations in both environmental and biological samples, indicating the utility of morphological control of AuNRs both in-the-field and at points-of-care.

Most of the aforementioned reports used spectrophotometers to study ensembles of AuNRs. Although some studies used TEM intermittently to verify nanorod morphology, this approach is limited in time resolution, and generally requires stopping reactions for analysis. Optical monitoring of reactions of single AuNRs can also be accomplished in situ. Dark-field spectral imaging has been used to study anisotropic etching of individual AuNRs by hydrogen peroxide, ${ }^{49}$ potassium iodide/iodine,${ }^{50}$ and gold(III). ${ }^{53}$ In a different experimental design, luminescence was employed to study the cyanide etching of AuNRs. ${ }^{47}$ Additionally, dissolution of AuNRs via substrate voltage tuning has been monitored using dark-field hyperspectral imaging. ${ }^{48}$ Perhaps the most commonly reported reagent for etching single AuNRs in recent years is iron(III) chloride, starting with bulk studies in 2009. ${ }^{82}$ Since then, $\mathrm{FeCl}_{3}$ etching of single AuNRs 
has been reported using dark-field monitoring, sometimes utilizing Le Châtlier's principle to drive the reaction. ${ }^{51,52}$ Ferric etching of single AuNRs using an electron beam, monitored by liquid-TEM, has also been reported. ${ }^{54}$ Excepting the electron beam study, these reports evoked purely chemical mechanisms to explain their reported chemistries. However, the light-induced etching of $\mathrm{AuNRs}$ using $\mathrm{FeCl}_{3}$ has also been reported, both in bulk studies, ${ }^{83}$ and single AuNR experiments using one-photon luminescence. ${ }^{46}$ Due to significant interest in ferric etching of AuNRs and the intriguing mechanistic parameter space, $\mathrm{FeCl}_{3}$ was employed for etching in this report.

\section{Single AuNR Reactions:}

After single AuNRs deposited in the microbubble resonator were identified, they were chemically etched using $\mathrm{FeCl}_{3}$. The etching solution, ranging between $250 \mu \mathrm{M}$ and $2 \mathrm{mM} \mathrm{FeCl}$ dissolved in dilute hydrochloric acid ( $\mathrm{pH} 1.3$ ) to prevent hydrolysis of the oxidant, was flowed into the microbubble. Due to differences in sensitivity resulting from microbubble geometries, mode selection, and even nanorod location within the same microbubble, the relative photothermal signals between nanorods cannot be directly compared. However, the relative signal of one AuNR reacting over time, using the same resonance, directly maps onto a change in absorption cross-section of the nanorod at the pump wavelength, and thus its etching progress. Conveniently, nanorod etching was found to be photo-activated by the pump beam illumination (discussed further below), allowing controlled reaction initiation. The AuNRs were monitored by repeatedly rotating the linearly polarized pump, interrogating the relative absorption and orientation of the AuNR as it is etched. Importantly, before each polarization trace is taken, a beam-centering algorithm is used to mitigate any false signal decrease from spatial drift of the bubble. The centering also serves as a "dosing" period to enable AuNR etching between polarization traces.

Figure 4A (i-iii) features three exemplary traces of a single AuNR reaction (additional examples in the Supporting Information). These three reactions were taken in different microbubbles on different days, confirming reproducibility of the experiment. A logarithmic version of reaction (i) (Figure 4B) readily shows the late stage continued reaction progression along with AuNR rotation. This behavior is better illustrated in the extracted maximum signal and angle traces from reaction (ii) seen in Figure $\mathbf{4 C}$ and Figure 4D respectively. The reactions slowed as they progressed, as seen by the plateauing effect in the maximum signal. This plateauing is a direct result of the photo-activation mechanism: as a nanorod shrinks, its absorption cross-section decreases, resulting in less light absorption and thus slower etching. This photoactivation is further confirmed by incrementally increasing the pump power (dotted vertical lines), quickening the reactivity before plateauing once again. Although the three reactions in Figure 4A were taken at ferric chloride concentrations spanning almost an order of magnitude, the time scales of reaction vary by much less (discussion in Supporting Information). It is also evident that AuNRs sometimes rotate as they etch, especially late in reactions, as seen in Figure 4D and discussed later (see Single AuNR Rotations).

A control experiment was performed with a nanorod-containing resonator filled with dilute hydrochloric acid ( $\mathrm{pH} 1.3$ ) without $\mathrm{FeCl}_{3}$, confirming that the acid alone is not enough to etch the nanorods under illumination. Additionally, when nanorods are left in etching solution for multiple days without laser illumination, they do not observably react, supporting a photo-activated etching mechanism. AuNRs exposed to etching solution for hours within a microbubble, with the probe beam on and locked to waterdominated modes but no pump beam, also did not undergo significant etching, indicating that the probe beam is not sufficient to drive etching. Therefore, we hypothesize a photo-activated mechanism resulting from hot electrons generated from LPB decay (see Mechanistic Discussion). We also note that, occasionally, nanorods were "impervious" to photo-activated etching, as discussed further in the

\section{Supporting Information.}


To exemplify the spectroscopic versatility of this platform, nanorod etching was also induced with the $532 \mathrm{~nm}$ pump beam. Because this wavelength pumps the TPB (as well as direct interband transitions), nanorod orientation can still be tracked. In Figure 5A, a single AuNR reaction time series is shown for 532 nm-driven (TPB-driven) conditions, with a logarithmic version of the data shown in Figure 5B for clear visualization of late-stage etching data. The polarization traces are conspicuously different than in Figure 4 because of the presence of polarization-independent interband transitions. In Figure 5C, maximum signals are extracted for this reaction and one other TPB-driven reaction in the same resonator. For direct comparison, a different nanorod in the same microbubble was reacted using the $635 \mathrm{~nm}$ pump beam (LPB-

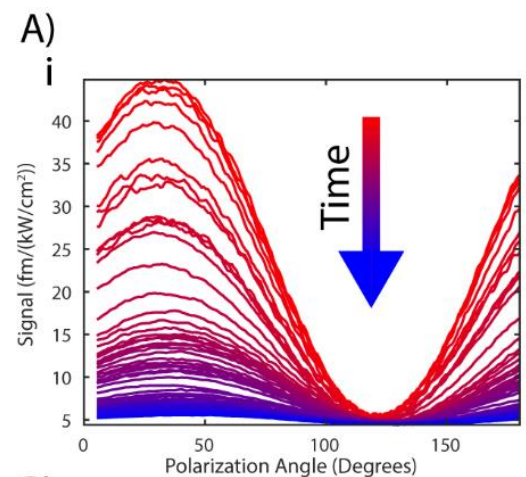

B)

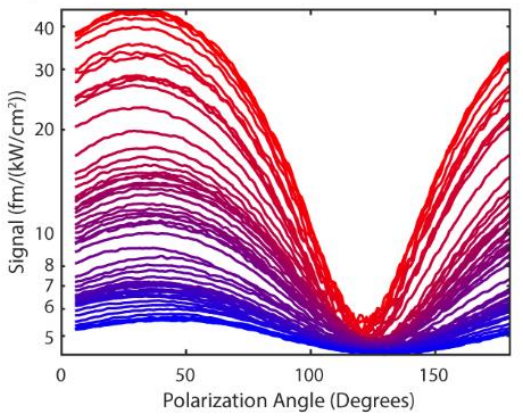

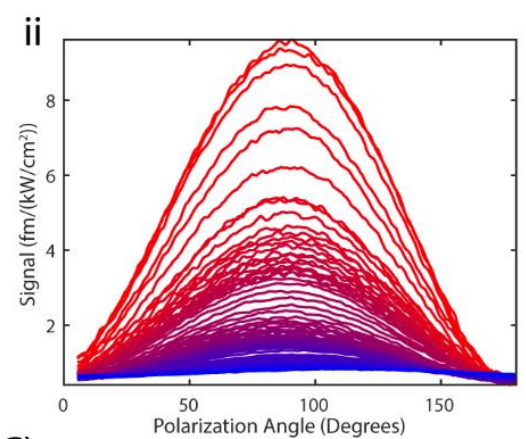

C)

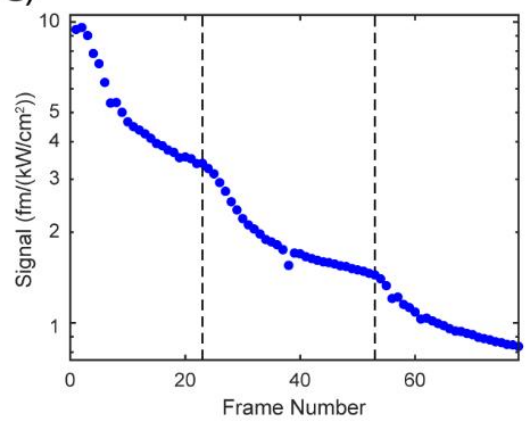

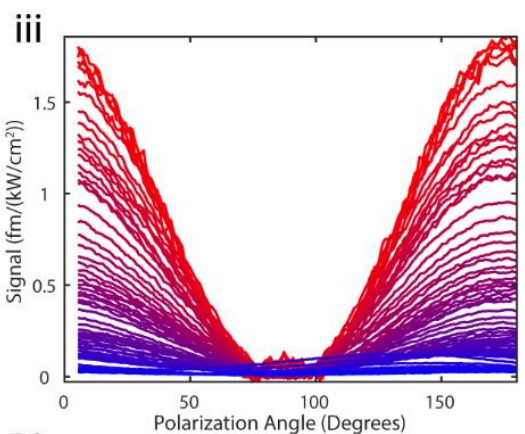

D)

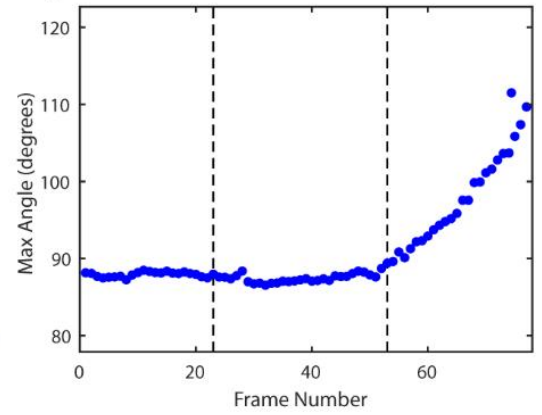

Figure 4. Etching single AuNRs. (A) Reaction series of polarization traces for three difference reactions, progressing in time from red traces to blue traces. Maximum signal is normalized by pump flux. (B) The data for reaction (ii), but with signal shown logarithmically. (C) Maximum signal of polarization traces over the course of reaction (ii), showing the decrease in relative absorption cross section at $635 \mathrm{~nm}$. Dashed lines indicate points in time at which pump power was increased. (D) Maximum angle of polarization traces over the course of reaction (ii), showing nanorod orientation. Dashed lines indicate points in time at which pump power was increased. Reaction conditions: dilute aqueous $\mathrm{HCl}$ ( $\mathrm{pH} 1.3$ ), room temperature, varied $\mathrm{FeCl}_{3}$ concentrations (i) $1 \mathrm{mM}$, (ii) 250uM, (iii) $2 \mathrm{mM}$. Pump fluxes for reaction (i) were $2.7,6.7,11.4,21.0$, and $34.5 \mathrm{~kW} / \mathrm{cm}^{2}$. Pump fluxes for reaction (ii) were $4.1,11.9,35.4 \mathrm{~kW} / \mathrm{cm}^{2}$. Pump fluxes for reaction (iii) were $6.4,15.9,31.6,57.3,121 \mathrm{~kW} / \mathrm{cm}^{2}$.

driven), with the extracted maximum signals shown in Figure 5D (note, this is the same reaction shown in Figure 4A (iii)). Overall, three such reactions were performed for each color in the same resonator to confirm reproducibility (Supporting Information). Although quantitative comparison of reaction rates between experiments is difficult due to the decreasing rate as absorption cross-section diminishes, it is clear from the extracted maximum signals that while the etching rate of the $532 \mathrm{~nm}$-induced reactions is faster than the rate of the $635 \mathrm{~nm}$-induced reactions, it is not multiple orders of magnitude faster, in contrast to previously reported bulk measurements, discussed further below. ${ }^{83}$ The shapes of maximum signal traces for the LPB-driven and TPB-driven reactions are noticeably different, with the LPB-driven reactions yielding a concave up shape, and the TPB-driven reactions yielding a concave-down (Figure 5c (i)) or even 
A)

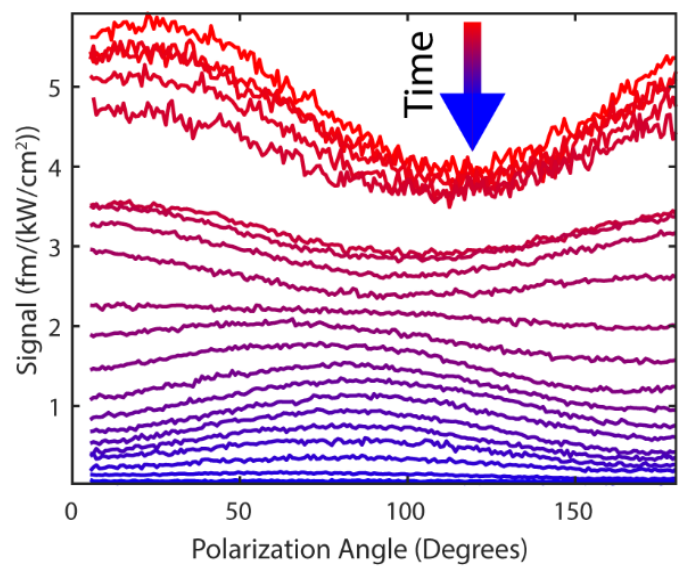

C)

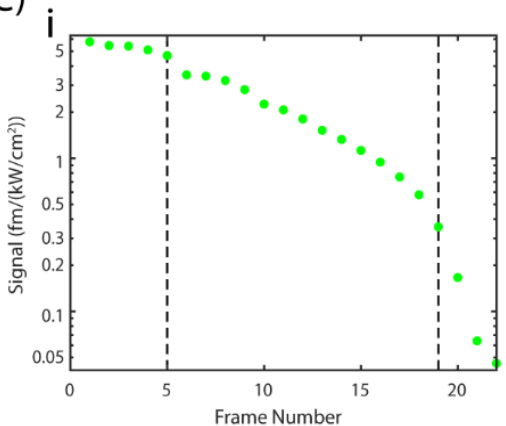

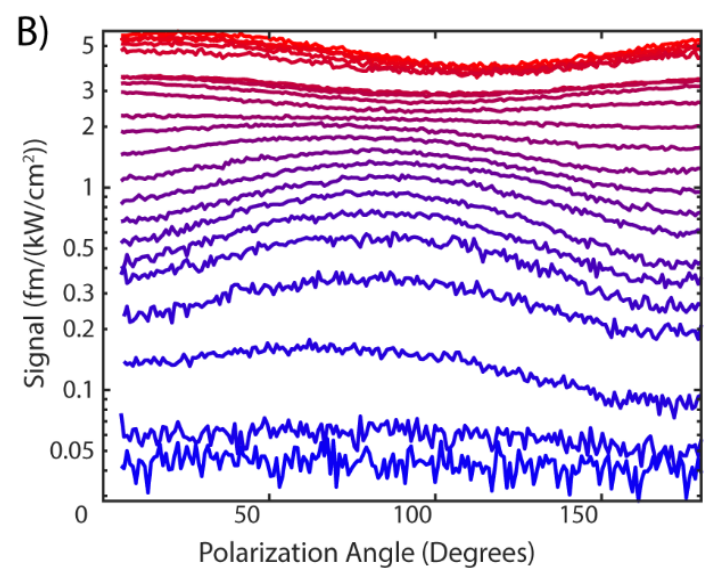

D)
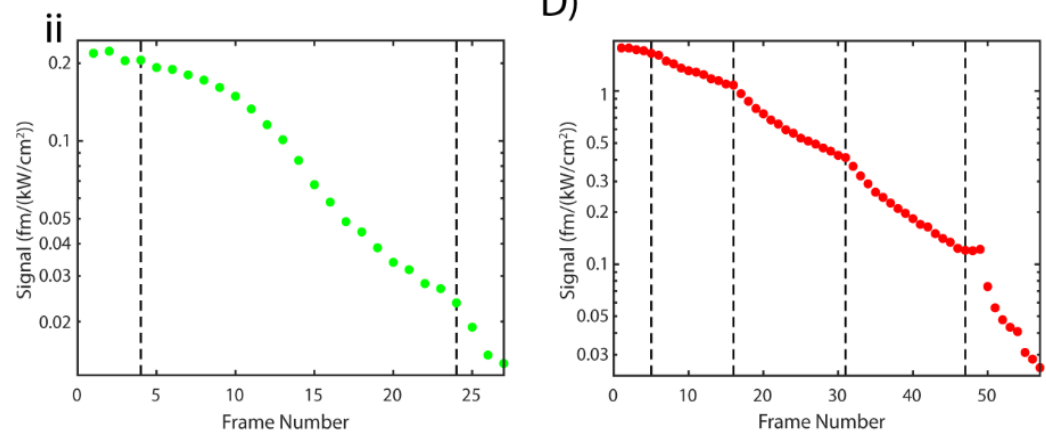

Figure 5. Etching reactions driven at two different pump wavelengths. (A) The reaction of a single AuNR being driven with the $532 \mathrm{~nm}$ pump beam, progressing in time from red traces to blue traces. Maximum signal is normalized by pump flux. (B) The same data as in (A), but with signal shown logarithmically. (C) (i) Maximum signal of polarization traces for the reaction shown in (A). (ii) A similar trace for a different nanorod reacted in the same bubble using the $532 \mathrm{~nm}$ beam. Dashed lines indicate time points at which pump power was increased. (D) Maximum signal of polarization traces for the reaction of a nanorod in the same bubble, but using the $635 \mathrm{~nm}$ pump beam to drive the reaction. Dashed lines indicate time points at which pump power was increased. Reaction conditions: dilute aqueous $\mathrm{HCl}\left(\mathrm{pH}\right.$ 1.3), room temperature, $1 \mathrm{mM} \mathrm{FeCl}_{3}$. Pump fluxes for reaction $(\mathrm{Ci}) 16.1,39.2,63.3 \mathrm{~kW} / \mathrm{cm}^{2}$. Pump fluxes for reaction (Cii) were $6.4,31.6,57.3 \mathrm{~kW} / \mathrm{cm}^{2}$. Pump fluxes for reaction (D) were $6.4,15.9,31.6,57.3,121 \mathrm{~kW} / \mathrm{cm}^{2}$.

sigmoidal shape (Figure 5c (ii)). With the above data in mind, the hypothesized mechanism of photoactivated etching in these experiments will now be discussed.

\section{Mechanistic Discussion:}

For many reported etching reagents, a simple redox analysis predicts that AuNR etching should not be spontaneous. Though the oxidation potential for gold nanorods can be significantly lower than that of bulk gold ${ }^{84}$ it has nonetheless been demonstrated that additional reagents are typically required to modulate reactivity. For example, CTAB at concentrations above the critical micelle concentration (CMC) facilitates $\mathrm{Au}(\mathrm{III})$ etching of AuNRs, presumably by complexing with $\mathrm{Au}(\mathrm{III})$ and preferentially aggregating near AuNR tips. ${ }^{85} \mathrm{CTAB}$ dependence was also observed in the first report of $\mathrm{FeCl}_{3}$ etching of AuNRs, including at $\mathrm{CTAB}$ concentrations well below the $\mathrm{CMC}$, and was hypothesized to be due to the complexation of bromide from $\mathrm{CTAB}$ and chloride from $\mathrm{FeCl}_{3}$ with gold, lowering its oxidation potential. ${ }^{82}$ In the single AuNR reactions in this report, oxidant concentration is two orders of magnitude lower than bulk studies showing light-independent etching (Supporting Information). This means that any halide dependence from $\mathrm{CTAB}$ or $\mathrm{FeCl}_{3}$ is negligible in comparison to the much larger chloride concentration from the dilute 


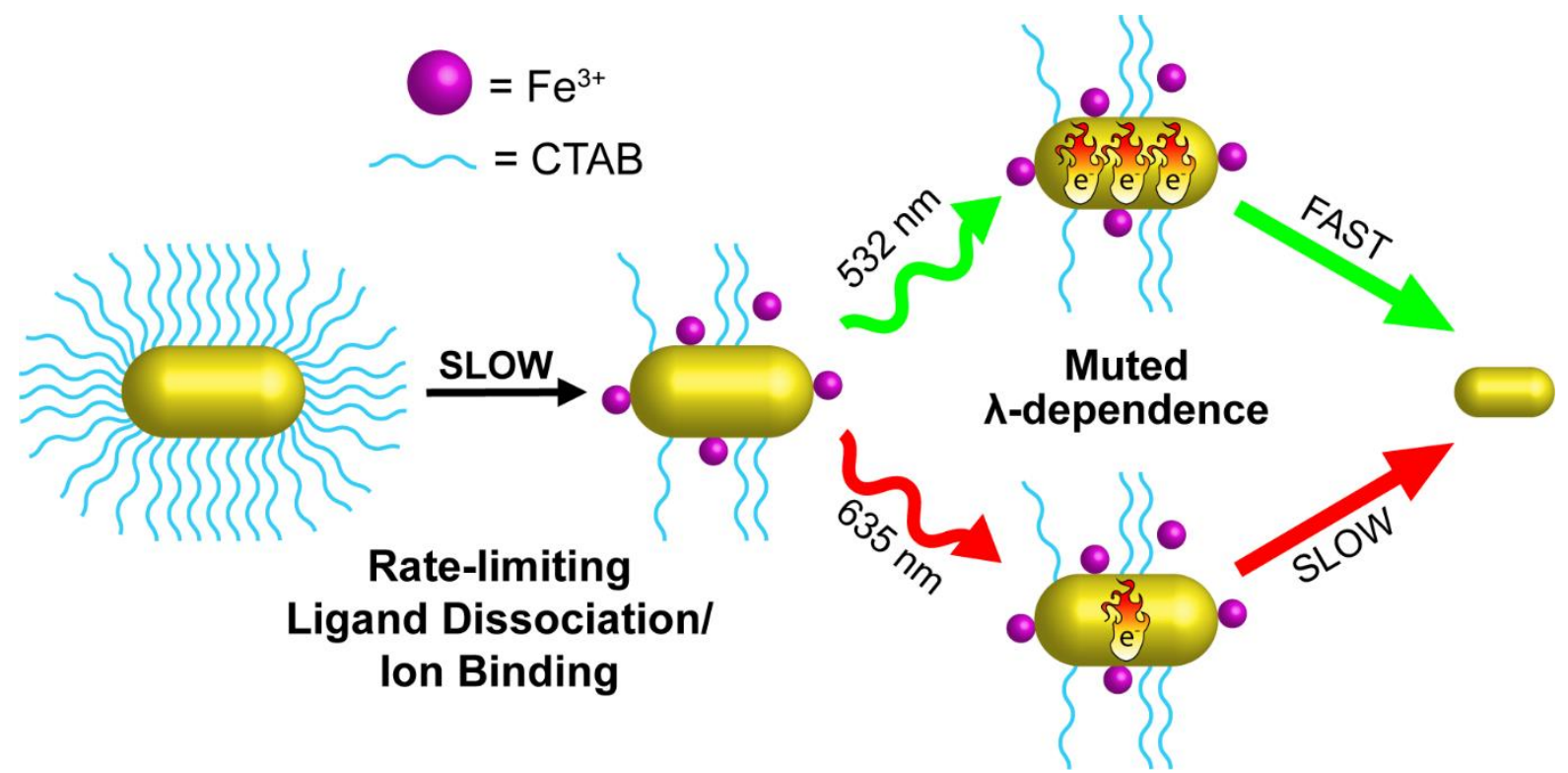

Figure 6. Proposed mechanistic explanation for etching rates. A slow initial step requiring CTAB dissociation before ferric ions can bind determines the overall rate for the reaction, muting the effect of the higher rate for TPB excitation, even though hot electrons are more efficiently generated. Eventually, etching stops when the absorbed light falls below a threshold necessary for hot-electron-driven etching.

hydrochloric acid. In conjunction with the studies discussed below, it is evident that CTAB plays an important role beyond halide donation.

In the bulk study by Toste and co-workers, ${ }^{83}$ etching was multiple orders of magnitude faster when driven with interband excitation than with LPB excitation, a phenomenon attributed to differences in the generated hot electron distributions. This chemistry was accomplished with CTAB concentrations above the CMC. In contrast, in the single AuNR etching reported by Khatua and co-workers, ${ }^{46} \mathrm{CTAB}$ was thoroughly stripped from the surface of the nanorods using ozone. Using only a green laser to drive etching, which excites both the TPB and interband transitions, the authors then exhibited bidirectional control over the AuNR aspect ratio, attributed to a balance of thermally driven and hot electron driven mechanisms. In our work, CTAB was removed from solution and reduced at the surface of deposited nanorods by flowing water following deposition (Methods). However, due to the gentle nature of this approach, we speculate that our conditions are at an intermediate surface $\mathrm{CTAB}$ concentration between reports from Khatua and Toste. We hypothesize that this intermediate CTAB concentration results in a hot electron etching mechanism that is different than in the above reports.

Interest in hot carrier chemical processes has exploded in recent years in a variety of applications, ${ }^{86}$ especially photocatalysis, ${ }^{87-89}$ with a particular emphasis on harvesting solar energy for driving chemical reactions. The mechanisms of hot carrier generation and transfer have been extensively studied, ${ }^{90-92}$ including the hot carrier energy distribution's dependence upon excitation wavelength. ${ }^{93}$ Notably, significant efforts have been made towards untangling the contributions of hot carrier effects and photothermal effects in both nanoparticle synthesis ${ }^{94}$ and plasmonic photocatalysis, ${ }^{95}$ as well as mapping hot carrier driven catalytic reactions on the surfaces of nanorods. ${ }^{96,97}$ Although there are still significant unresolved questions in understanding plasmon-driven chemistry, the mechanisms of hot carrier transfer can generally be predicted by modeling the energy overlap between the hot carrier, the molecular orbitals of the adsorbed molecule/atom, and the fermi level of the metal. ${ }^{98}$

Hot carriers likely play a dominant role in the chemical etching of AuNRs in the microbubble. With a temperature rise at the nanorod's surface expected to be under $1 \mathrm{~K},{ }^{46}$ the observed etching mechanism should not be significantly influenced by photothermal heating, as with the higher power reactions in 
Khatua's report. ${ }^{46}$ With no noticeable dark reaction rate, and a photothermal mechanism ruled out, a hot carrier mechanism, whereby the decay of LSPRs or excitation of interband transitions result in hot electrons that can transfer to ferric ions on the nanorod surface, must be invoked to explain the observed reactions. Hot electrons, which have been driven out of thermal equilibrium to a higher energy than their ground state, lower the Gibbs free energy of the etching reaction, ${ }^{52}$ favoring products. The change in energy level of reactants also reduces the thermal activation barrier for electron transfer to ferric ions, modifying the forward rate constant and thus the reaction kinetics. ${ }^{95}$ Specifically, photoexcitation of gold nanoparticles has been shown to lower the activation enthalpy for transferring electrons from gold nanoparticles to $\mathrm{Fe}^{3+}$, and the dependence of this process upon excitation wavelength and power has been studied. ${ }^{99}$

When the LSPRs of an AuNR decay, hot electrons are generated from the conduction band of the nanorod. These hot electrons are on average at lower energy than those generated by interband transitions, though a few carriers will be hotter in the LSPR decay case. ${ }^{83}$ Toste and coworkers found that the larger population of lower-energy hot electrons generated from interband transitions drove ferric chloride etching of AuNRs orders of magnitude more efficiently than those generated from the LPB ${ }^{83}$ However, given that the TPB-driven reactions in this report proceed at a rate that is comparable to the rate of the LPB-driven reactions (Figure 5), we conclude that the etching mechanism observed here is fundamentally different than that observed by Toste. ${ }^{83}$ Ultimately, the reaction relies on ferric ions binding to the nanorod before hot electron transfer can take place. Thus, a plausible mechanism entails a two-step process, whereby slow ligand exchange of $\mathrm{CTAB}$ with $\mathrm{Fe}^{3+}$, or intercalation of the $\mathrm{Fe}^{3+}$ through the $\mathrm{CTAB}$, is followed by fast photoactivated etching. A relative increase in the rate of the photoactivated step is then ultimately masked in the observed rate due to the slowness of the first ligand exchange step, as shown Figure 6. In the limit where ligand exchange is slow, increases in the rate of the photo-induced step would give a somewhat muted effect on the overall reaction rate, as observed. Intriguingly, it has been shown with single silver nanoparticles that photoexcitation can promote weakening of ligand-nanoparticle bonds, thereby lowering the energy barrier for reactions at the nanoparticle surface. ${ }^{100}$ If such an effect exists for CTAB-coated AuNRs, it might also be dependent upon excitation-wavelength, resulting in differences in the observed kinetics of LPB-driven and TPB-driven reactions.

To further understand the shape of the reaction profiles, we modeled the spectral changes of the TPB and LPB for a nanorod being etched (Supporting Information). This simulation was done for a variety of etching schemes, from tip-only etching to side-only etching. Modelling was able to reproduce the concave-up shape observed in the LPB-driven reactions (Figure 4C). However, no combination of variables was able to capture the concave down or sigmoidal trend seen in some TPB- driven reactions (Figure 5Ci and ii), or fully reproduce the threshold behavior observed. Thus, with a completely linear reaction mechanism ruled out, we can speculate on possible origins of non-linearity in the etching mechanism. One possible origin of non-linearity stems from the evolution of nanorod morphology over time. For example, it was observed by Khatua that for certain laser powers, nanorod LPBs would red-shift, then blue-shift. Another possible origin derives from the changing concentration of CTAB, with a relatively dense coverage providing competitive inhibition for ferric ion binding at early times but AuNR etching resulting in easier access at later times. Future studies utilizing multiple pump beams could be valuable in studying these complex kinetics, as the evolution of the relative signal of the LPB and TPB excitation beams would yield important insight into the reaction mechanism. Thus, our new microbubble platform gives a path forward for spectroscopic mechanistic studies into the wavelength-dependence of hot-carrier driven chemical dynamics in single plasmonic nanoparticles.

\section{Single AuNR Rotations:}

Beyond using our microbubble spectrometer to monitor and control nanoparticle size, we can also

use it to monitor and control nanoparticle orientation, adding significant utility to the microbubble 
spectrometer platform. Indicated by the shift in peak polarization during etching reactions (Figure 4 and Figure 5), AuNRs can rotate while etching. Alternatively, active rotation can be induced with the pump beam, allowing for control over nanorod orientation. This control results from the optical torque exerted by linearly polarized light on an anisotropic, absorbing plasmonic structure (Figure 7a), a phenomenon that has been demonstrated experimentally ${ }^{101}$ and theoretically, ${ }^{102,103}$ and is discussed further in the Supporting Information.

Theory predicts that the optical torque acting on the AuNR from the $635 \mathrm{~nm}$ pump beam will align the AuNR perpendicular to the polarization of the incident beam. Indeed, this perpendicular alignment is exhibited upon sufficient excitation power. In this way nanorod orientation can be controlled to within approximately 10 degrees (Figure 7b) as AuNR orientation is stepped through a $\sim 180$ degree rotation. This control was accomplished by monitoring the photothermal signal, dislodging the nanorod with a large optical torque above some threshold incident power, and dithering the polarization until the photothermal signal was minimized at the desired polarization angle. This thresholding behavior was demonstrated further by a stepwise ramping of the pump laser intensity, resulting in an upward staircase of photothermal signal, until rotation was finally induced. As can be seen in the examples in Figure 7C, two different nanorods required significantly different pump powers to dislodge them from the microbubble surface.

A)
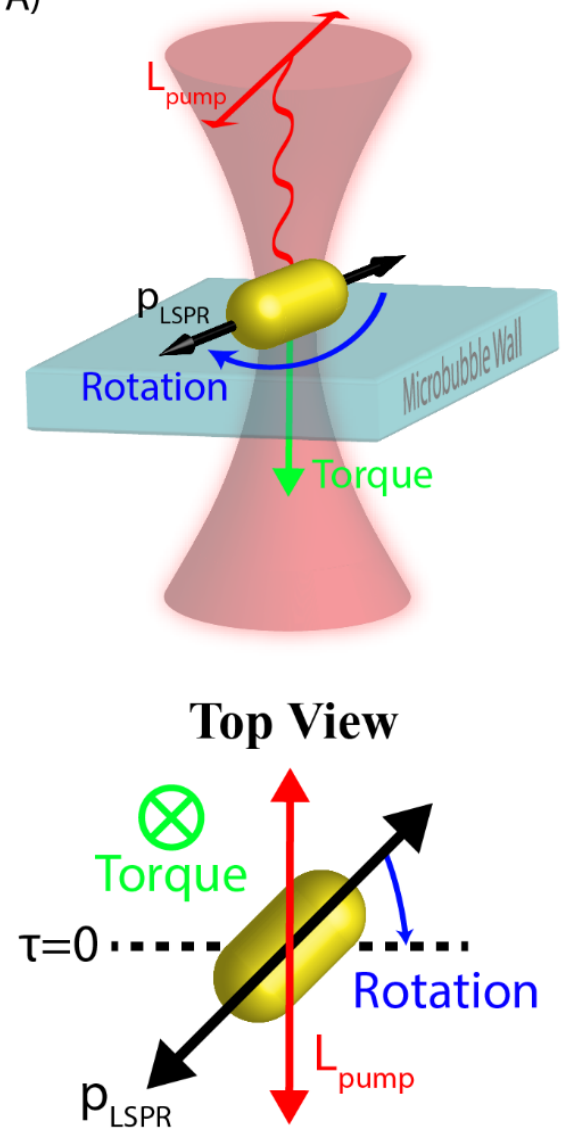

B)

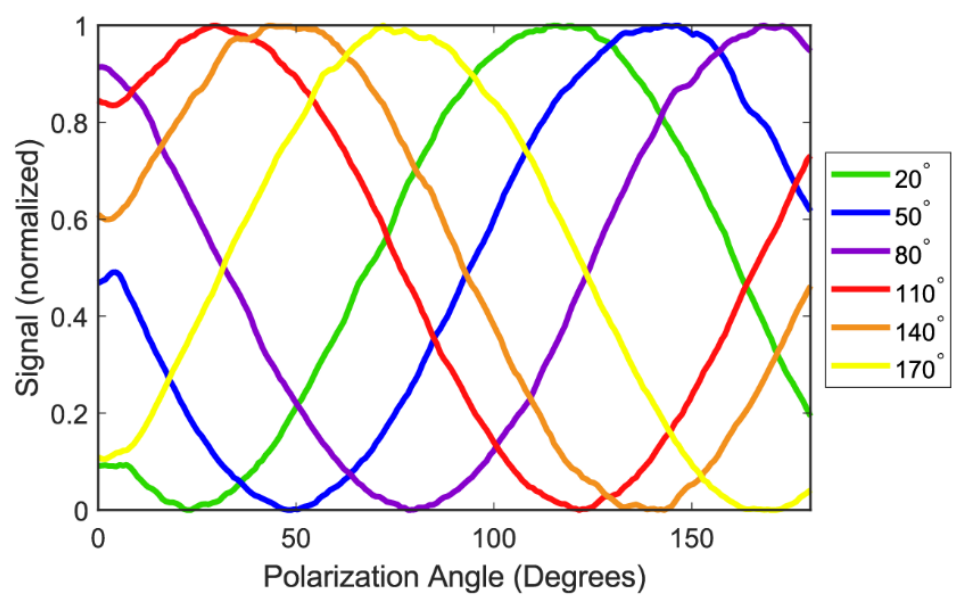

C)

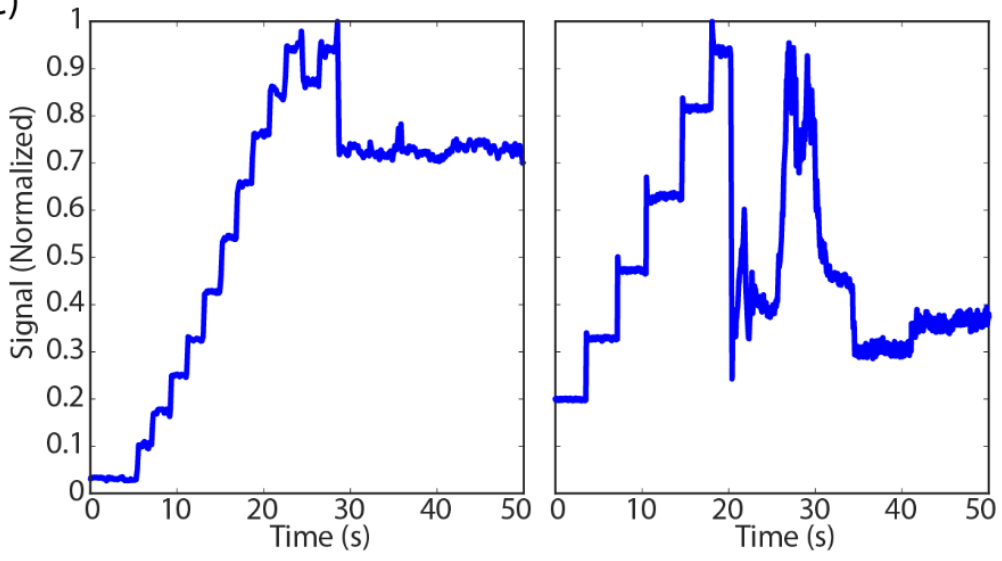

Figure 7. Orientation control of single AuNRs. (A) A cartoon illustrating the optically induced torque that a AuNR experiences under illumination with linearly polarized light, both from side-view (top) and top-view (bottom). (B) A series of pumping experiments showing optical control of nanorod orientation. (C) Trace showing the photothermal signal as two different AuNRs are pumped at increasing laser powers until the AuNRs dislodge slightly from the resonator wall and rotate, eventually settling down off-axis of the polarization. 
Following dislodgment, the signal quickly stabilized to around $70 \%$ of the maximum signal for the left nanorod trace, whereas it behaved semi-stochastically for the right trace, before settling at $<40 \%$ of the maximum signal. These differences in orientational dynamics highlight the differences in the local environments around the two nanorods, including both Coulombic effects and refractive index differences. These staircase experiments were performed repeatedly for both nanorods in Figure $\mathbf{7 C}$ to confirm reproducibility (Supporting Information).

Light-induced rotation during reactions was observed more frequently as AuNR etching progressed. Likely, as Coulombic attractions between the AuNR and the resonator's surface were weakened, hydrodynamic or optical torques were allowed to rotate the AuNR. Although rotation events could be forced in water-filled resonators, higher pump thresholds were generally required, and AuNRs were immune to rotation at powers that would result in rotation in ferric chloride solution. Therefore, it appears that the presence of etchant reduces the Coulombic attraction between nanorods and the resonator wall, possibly through charge screening, permitting facile rotation. Though optical rotation of nanorods has been seen in previous experiments, the coupling between evolving surface chemistry and propensity for rotation has not been explored to our knowledge.

Radiation pressure and optical gradient forces could also influence AuNRs, affecting the rotation power threshold. However, varying the pump beam focus position, which would change forces along the optical axis, did not significantly impact rotation thresholds. Therefore, it appears that the torque described above is the dominant driver of nanorod rotation. Although scattering forces in three-dimensional trapping can orient anisotropic plasmonic nanoparticles parallel to the optical axis of the excitation beam, ${ }^{104}$ one would expect this to result in a highly stochastic signal over time in the polarization traces of Figure 3 and Figure 4, as well as a revival of signal upon shutting off and turning back on the pump beam. Such behavior was not observed. Therefore, AuNRs likely remain parallel to the plane of the resonator's surface during rotation, rotating only in two dimensions.

\section{Conclusions and Outlook:}

We have demonstrated microbubble resonators as a robust platform for studying chemical dynamics in solution via single-particle absorption spectroscopy. We used a microbubble spectrometer to observe changes in the optical properties of AuNRs as they were controllably etched by ferric chloride via a photo-induced mechanism. Additionally, we monitored and controlled the orientation of the AuNRs using optical torque. With this demonstration, we lay the groundwork for studying more complex reaction dynamics of single particles and molecules. Thus, this technique provides a complementary measurement to the luminescence and dark-field methods previously used to observe similar reactions as reported here. In particular, the demonstrated exquisite sensitivity offers prospects of examining non-emissive objects inaccessible with fluorescence and too small to observe with scattering, which scales as $1 / \mathrm{Volume}^{2}$, a more severe penalty than in absorption measurements, which scale more favorably as $1 /$ Volume. Furthermore, rotational control could be used to estimate Coulombic forces attaching deposited objects to the resonator, helping to understand the interface between nanoparticles and the surface. This knowledge, combined with structured light field manipulation of nanoparticles, ${ }^{105}$ might be used to arrange arrays of plasmonic nanoparticles as desired. We believe that optical control of plasmonic nanoparticles within a microbubble resonator will yield new paths for in-solution, photonic-plasmonic assembly, and live-control of emergent optical properties in such coupled systems. Additionally, by providing a direct thermal readout, our method could be used to untangle the respective contributions of photothermal heating and hot carrier generation for nanoparticle reactions, aiding the design of improved nanocatalysts. Overall, there is a compelling case for the use of microbubbles in materials studies, sensing, and chemical kinetics, and even hybridizing them with plasmonic or acoustic sensing schemes for further applications. Microbubble absorption spectrometers thus hold great potential for pushing the frontiers of absorption spectroscopy at the nanoscale. 


\section{Methods:}

Microbubble fabrication: Microbubble resonators were fabricated according to the method reported by Yang and coworkers. ${ }^{106}$ First, a glass capillary (Polymicro Technologies, TSP250350) is tapered using a heat-and-pull method, until it is approximately $25 \mathrm{um}$ in diameter. Next, counter-propagating $\mathrm{CO}_{2}$ laser beams are focused onto the capillary while positive pressure is applied from the inside of the capillary using an inert gas. The heat from the laser beams softens the capillary, allowing for the local expansion of the capillary to 50-100 um in diameter, depending on the experimental parameters. Eventually, radiative cooling from the expanded glass outcompetes the expansion process, and the bubble's size stops increasing. To operate in the quasi-droplet regime, a wall thickness close to the wavelength of the laser beam used for WGM excitation is desirable. Microbubble wall thickness is determined by an equation reported by Henze and coworkers, ${ }^{107}$ and validated separately by others. ${ }^{108}$

Tapered optical fiber fabrication: Single-mode optical fiber was purchased from Corning (HI 780C). Tapered fibers are made by removing the polymer sheath, cleaning the fiber, and tapering using a heat-andpull method with a hydrogen torch and motorized actuators (Thorlabs Z825B) until the fiber returned to single-mode, as determined using a 785nm diode laser (Thorlabs LPS-785-FC) and optical power meter.

Instrumentation for photothermal spectroscopy: A tunable, ultra-narrow linewidth, fiber-coupled CW laser (Newport TLB-6712) with a wavelength range of 765-781 nm was used for coupling into resonators. Pound-Drever-Hall locking electronics were constructed as previously reported, ${ }^{13}$ except for the use of a different voltage-controlled oscillator (Mini Circuits ZX95-310A+) and different lithium niobate phasemodulator (EOSPACE PM-0S5-01-PFA-PFA-765/782). PDH feedback was applied to the tunable laser using high-speed servo controller (Newport LB1005). The optical output from the experiment was collected using an APD (Thorlabs APD430A), and the photothermal signal was extracted using a lock-in amplifier (Ametek 7265). The resulting signal was collected using a Data Acquisition (DAQ) card (National Instruments BNC2120) for later processing. Custom LabVIEW code was used for instrumentation control. For photothermal mapping, a lock-in time constant of $20 \mathrm{~ms}$ or $50 \mathrm{~ms}$ was used. For polarization traces, a lock-in time constant of $50 \mathrm{~ms}$ was used.

Diode lasers were used for pump beams, with the wavelengths 532nm (FTEC2 532-20), $635 \mathrm{~nm}$ (FTEC2 635-50), and $785 \mathrm{~nm}$ (Thorlabs LPS-785-FC). The pump beam was amplitude modulated using an optical chopper system (Thorlabs MC200B), and steered using galvanometer mirrors (Thorlabs GVS212) run by outputs from the DAQ mentioned above, modified using custom electronics. The pump beam was focused using a piezo-controlled (Thorlabs DRV517) objective (Nikon Plan 40x, 0.65 NA). Pump beam polarization was controlled using a three-optic system, comprised of a linear polarizer (LPVISE100-A), followed by a liquid crystal variable retarder (Thorlabs LCC1423-A, LCC25) with its fast axis set 45 degrees relative to the polarization axis, followed by a zero-order achromatic quarter-wave plate (Thorlabs AQWP05M-600) with its fast axis set 45 degrees relative to the liquid crystal's fast axis. In this design, tuning of the liquid crystal voltage results in a rotation of linearly polarized light at the output of the threeoptic system.

Polarization Plots: Shown in Figure 3C and Figure 3D, AuNRs are first identified by photothermally mapping them at different pump polarizations, and processing these maps with a 2D-Gaussian fit, which results in background subtraction. Then, the maximum signals for each plot are together fit to provide a depth-of-modulation $(\mathrm{M})$, using equation (1), which also gives the maximum signal $\left(\sigma_{\max }\right)$ plotted in Figure $4 \mathrm{C}$, and the polarization angle of the maximum signal $\left(\theta_{\max }\right)$ plotted in Figure 4D. 


$$
\sigma(\theta)=\sigma_{\max }\left(1-\mathrm{M} * \sin ^{2}\left(\theta-\theta_{\max }\right)\right)
$$

For the data in Figure 3E, the liquid crystal is used to rapidly collect many data points that are then fit to equation (1) to obtain M. This method does not include background subtraction.

Bulk/UV-Vis Studies: The bulk absorption spectrum in Figure 3B was taken using a UV-Visible Spectrophotometer (Varian Cary 50). Additionally, studies were conducted to confirm the effects of CTAB concentration and $\mathrm{FeCl}_{3}$ concentration on bulk nanorod etching. The effects of added $\mathrm{NaCl}$ were studied to further examine the impacts of chloride concentration. Results and further discussion are in the Supporting Information.

Nanorod deposition in microbubbles: All chemicals were purchased through Sigma-Aldrich unless otherwise noted. To deposit nanorods in a microbubble, a 500x serial dilution is made of AuNRs (Nanopartz A12-40-650-CTAB-DIH-1-25) in a solution of 200x diluted $\mathrm{HCl}$ and $25 \mathrm{uM} \mathrm{CTAB}$ in water. The low $\mathrm{CTAB}$ concentration prevents nanorod aggregation during deposition, but keeps the CTAB concentration well below the CMC of $\sim 1 \mathrm{mM}$. Dilute hydrochloric acid, which results in a $\mathrm{pH}$ of around 1.3, encourages binding of the nanorods on the resonator interior by enhancing Coulombic interactions. ${ }^{9}$ For deposition, water is first flowed through the resonator using a syringe pump attached to the first port of the resonator's capillary. Then, dilute $\mathrm{HCl}$ is flowed through the resonator to prime the glass surface for deposition. Next, deposition solution is backfilled through the second capillary port, which is cut to a much shorter length to reduce deposition of AuNRs to the capillary's interior walls. Following this, dilute $\mathrm{HCl}$ is flowed through the resonator, followed by water, through the first port of the capillary to push out the deposition solution while maintaining a $\mathrm{pH}$ gradient. Water is flowed through the resonator for at least several minutes to ensure the removal of non-bound objects, and remove excess CTAB from the nanorod surfaces.

Reactions in Microbubbles: All chemicals were purchased from Sigma-Aldrich. Reaction mixtures are made by dissolving and serial diluting ferric chloride hexahydrate in 1/200 dilute hydrochloric acid, resulting in a solution $\mathrm{pH}$ of around 1.3. While reaction solution was flowed into the bubble, resonances shift as the refractive index being probed by the WGM changed. Complete filling of the microbubble with reaction mixture is indicated when the resonances have stopped shifting. Following this stabilization, the syringe pump pressure is released, resulting in a microbubble primed for etching experiments.

Acknowledgements: RHG acknowledges support from NIH (GM127957) and NSF (DBI-1556241). SNC acknowledges support from the Okinawa Institute of Science and Technology Graduate University. We thank J. Millstone and C. Murphy for enlightening conversations about nanoparticle chemistry.

\section{Supporting Information Available:}

- Geometric parameters of microbubbles, COMSOL simulations, comparison with microtoroids, static offset vs. modulated signal, mode shifting for different $d n / d T$ values (experimental), modeling of LPB and TPB during etching, thermal expansion, diagram for mode indices, additional single AuNR etching data, additional rotation data, bulk reaction results, brief discussion on extracting reaction kinetics, background on AuNR etchants, impervious nanorods, theory of nanorod rotation, Matlab code for modeling plasmon changes during etching.

\section{References:}

1. Heylman, K. D.; Knapper, K. A.; Horak, E. H.; Rea, M. T.; Vanga, S. K.; Goldsmith, R. H. Optical Microresonators for Sensing and Transduction: A Materials Perspective. Adv. Mater. 2017, 29, 1700037. 
2. Kim, E.; Baaske, M. D.; Vollmer, F. Towards next-generation label-free biosensors: recent advances in whispering gallery mode sensors. Lab Chip 2017, 17, 1190-1205.

3. Bozzola, A.; Perotto, S.; De Angelis, F. Hybrid plasmonic-photonic whispering gallery mode resonators for sensing: a critical review. Analyst 2017, 142, 883-898.

4. Li, Y.; Jiang, X.; Zhao, G.; Yang, L., Whispering gallery mode microresonator for nonlinear optics. In arXiv e-prints, https://arxiv.org/abs/1809.04878, 2018. Accessed September 13 ${ }^{\text {th }}, 2018$.

5. Wade, J. H.; Bailey, R. C. Applications of Optical Microcavity Resonators in Analytical Chemistry. Annu. Rev. Phys. Chem., Vol 9 2016, 9, 1-25.

6. Swaim, J. D.; Knittel, J.; Bowen, W. P. Detection of nanoparticles with a frequency locked whispering gallery mode microresonator. Appl. Phys. Lett. 2013, 102, 183106.

7. Zhi, Y. Y.; Yu, X. C.; Gong, Q. H.; Yang, L.; Xiao, Y. F. Single Nanoparticle Detection Using Optical Microcavities. Adv. Mater. 2017, 29, 1604920.

8. Kim, E.; Baaske, M. D.; Schuldes, I.; Wilsch, P. S.; Vollmer, F. Label-free optical detection of single enzyme-reactant reactions and associated conformational changes. Science Advances 2017, 3, e1603044.

9. Baaske, M. D.; Foreman, M. R.; Vollmer, F. Single-molecule nucleic acid interactions monitored on a label-free microcavity biosensor platform. Nature Nanotech. 2014, 9, 933-939.

10. Dantham, V. R.; Holler, S.; Barbre, C.; Keng, D.; Kolchenko, V.; Arnold, S., Label-Free Detection of Single Protein Using a Nanoplasmonic-Photonic Hybrid Microcavity. Nano Letters 2013, 13, 3347-3351.

11. Yu, W. Y.; Jiang, W. C.; Lin, Q.; Lu, T., Cavity optomechanical spring sensing of single molecules. Nature Commun. 2016, 7, 12311.

12. Baaske, M. D.; Vollmer, F., Optical observation of single atomic ions interacting with plasmonic nanorods in aqueous solution. Nature Photon. 2016, 10, 733-739.

13. Heylman, K. D.; Thakkar, N.; Horak, E. H.; Quillin, S. C.; Cherqui, C.; Knapper, K. A.; Masiello, D. J.; Goldsmith, R. H., Optical microresonators as single-particle absorption spectrometers. Nature Photon. 2016, 10, 788-795.

14. Thakkar, N.; Rea, M. T.; Smith, K. C.; Heylman, K. D.; Quillin, S. C.; Knapper, K. A.; Horak, E. H.; Masiello, D. J.; Goldsmith, R. H., Sculpting Fano Resonances To Control Photonic-Plasmonic Hybridization. Nano Letters 2017, 17, 6927-6934.

15. Knapper, K. A.; Heylman, K. D.; Horak, E. H.; Goldsmith, R. H., Chip-Scale Fabrication of HighQ All-Glass Toroidal Microresonators for Single-Particle Label-Free Imaging. Adv. Mater. 2016, 28, 29452950.

16. Knapper, K. A.; Pan, F.; Rea, M. T.; Horak, E. H.; Rogers, J. D.; Goldsmith, R. H. Single-particle photothermal imaging via inverted excitation through high-Q all-glass toroidal microresonators. Opt. Express 2018, 26, 25020-25030.

17. Heylman, K. D.; Knapper, K. A.; Goldsmith, R. H. Photothermal Microscopy of Nonluminescent Single Particles Enabled by Optical Microresonators. J. Phys. Chem. Lett. 2014, 5, 1917-1923.

18. Horak, E. H.; Rea, M. T.; Heylman, K. D.; Gelbwaser-Klimovsky, D.; Saikin, S. K.; Thompson, B. J.; Kohler, D. D.; Knapper, K. A.; Wei, W.; Pan, F.; Gopalan, P.; Wright, J. C.; Aspuru-Guzik, A.; Goldsmith, R. H. Exploring Electronic Structure and Order in Polymers via Single-Particle Microresonator Spectroscopy. Nano. Lett. 2018, 18, 1600-1607.

19. Chen, H. J.; Shao, L.; Li, Q.; Wang, J. F. Gold nanorods and their plasmonic properties. Chem. Soc. Rev. 2013, 42, 2679-2724.

20. Dreaden, E. C.; Alkilany, A. M.; Huang, X. H.; Murphy, C. J.; El-Sayed, M. A. The golden age: gold nanoparticles for biomedicine. Chem. Soc. Rev. 2012, 41, 2740-2779.

21. Huang, X. H.; El-Sayed, I. H.; Qian, W.; El-Sayed, M. A. Cancer cell imaging and photothermal therapy in the near-infrared region by using gold nanorods. J. Am. Chem. Soc. 2006, 128, 2115-2120.

22. Yin, D. Y.; Li, X. L.; Ma, Y. Y.; Liu, Z. Targeted cancer imaging and photothermal therapy via monosaccharide-imprinted gold nanorods. Chem. Comm. 2017, 53, 6716-6719. 
23. Ali, M. R. K.; Wu, Y.; Ghosh, D.; Do, B. H.; Chen, K.; Dawson, M. R.; Fang, N.; Sulchek, T. A.; El-Sayed, M. A. Nuclear Membrane-Targeted Gold Nanoparticles Inhibit Cancer Cell Migration and Invasion. ACS Nano 2017, 11, 3716-3726.

24. Meeker, D. G.; Chen, J. Y.; Smeltzer, M. S. Could targeted, antibiotic-loaded gold nanoconstructs be a new magic bullet to fight infection? Nanomedicine 2016, 11, 2379-2382.

25. Cao, J.; Sun, T.; Grattan, K. T. V. Gold nanorod-based localized surface plasmon resonance biosensors: A review. Sens. Actuators B-Chem. 2014, 195, 332-351.

26. Taylor, A. B.; Zijlstra, P. Single-Molecule Plasmon Sensing: Current Status and Future Prospects. ACS Sensors 2017, 2, 1103-1122.

27. Lin, K. Q.; Yi, J.; Hu, S.; Liu, B. J.; Liu, J. Y.; Wang, X.; Ren, B. Size Effect on SERS of Gold Nanorods Demonstrated via Single Nanoparticle Spectroscopy. J. Phys. Chem. C 2016, 120, 20806-20813.

28. Gao, Z.; Burrows, N. D.; Valley, N. A.; Schatz, G. C.; Murphy, C. J.; Haynes, C. L. In solution SERS sensing using mesoporous silica-coated gold nanorods. Analyst 2016, 141, 5088-5095.

29. Khatua, S.; Paulo, P. M. R.; Yuan, H. F.; Gupta, A.; Zijlstra, P.; Orrit, M. Resonant Plasmonic Enhancement of Single-Molecule Fluorescence by Individual Gold Nanorods. ACS Nano 2014, 8, 44404449.

30. Nima, Z. A.; Alwbari, A. M.; Dantuluri, V.; Hamzah, R. N.; Sra, N.; Motwani, P.; Arnaoutakis, K.; Levy, R. A.; Bohliqa, A. F.; Nedosekin, D.; Zharov, V. P.; Makhoul, I.; Biris, A. S. Targeting nano drug delivery to cancer cells using tunable, multi-layer, silver-decorated gold nanorods. J. Appl. Toxicol. 2017, $37,1370-1378$.

31. Wang, F.; Li, C. H.; Chen, H. J.; Jiang, R. B.; Sun, L. D.; Li, Q.; Wang, J. F.; Yu, J. C.; Yan, C. H. Plasmonic Harvesting of Light Energy for Suzuki Coupling Reactions. J. Am. Chem. Soc. 2013, 135, 55885601.

32. Gole, A.; Murphy, C. J. Seed-mediated synthesis of gold nanorods: Role of the size and nature of the seed. Chem. Mater. 2004, 16, 3633-3640.

33. Baida, H.; Christofilos, D.; Maioli, P.; Crut, A.; Del Fatti, N.; Vallee, F. Surface plasmon resonance spectroscopy of single surfactant-stabilized gold nanoparticles. Eur. Phys. J. D 2011, 63, 293-299.

34. Li, Z. M.; Mao, W. Z.; Devadas, M. S.; Hartland, G. V. Absorption Spectroscopy of Single Optically Trapped Gold Nanorods. Nano. Lett. 2015, 15, 7731-7735.

35. Yorulmaz, M.; Nizzero, S.; Hoggard, A.; Wang, L. Y.; Cai, Y. Y.; Su, M. N.; Chang, W. S.; Link, S. Single-Particle Absorption Spectroscopy by Photothermal Contrast. Nano. Lett. 2015, 15, 3041-3047.

36. Berciaud, S.; Cognet, L.; Tamarat, P.; Lounis, B. Observation of intrinsic size effects in the optical response of individual gold nanoparticles. Nano. Lett. 2005, 5, 515-518.

37. Gaiduk, A.; Yorulmaz, M.; Ruijgrok, P. V.; Orrit, M. Room-Temperature Detection of a Single Molecule's Absorption by Photothermal Contrast. Science 2010, 330, 353-356.

38. Chien, M. H.; Brameshuber, M.; Rossboth, B. K.; Schutz, G. J.; Schmid, S. Single-molecule optical absorption imaging by nanomechanical photothermal sensing. Proc. Natl. Acad. Sci. U. S. A. 2018, 115, $11150-11155$.

39. Crut, A.; Maioli, P.; Del Fatti, N.; Vallee, F. Optical absorption and scattering spectroscopies of single nano-objects. Chem. Soc. Rev. 2014, 43, 3921-3956.

40. Zrimsek, A. B.; Wong, N. L.; Van Duyne, R. P. Single Molecule Surface-Enhanced Raman Spectroscopy: A Critical Analysis of the Bianalyte versus Isotopologue Proof. J. Phys. Chem. C 2016, 120, 5133-5142.

41. Young, G.; Kukura, P. Interferometric Scattering Microscopy. Annu. Rev. of Phys. Chem. 2019, 70, 301-322.

42. Celebrano, M.; Kukura, P.; Renn, A.; Sandoghdar, V. Single-molecule imaging by optical absorption. Nature Photon. 2011, 5, 95-98.

43. Chong, S. S.; Min, W.; Xie, X. S. Ground-State Depletion Microscopy: Detection Sensitivity of Single-Molecule Optical Absorption at Room Temperature. J. Phys. Chem. Lett. 2010, 1, 3316-3322.

44. Maley, A. M.; Lu, G. J.; Shapiro, M. G.; Corn, R. M. Characterizing Single Polymeric and Protein Nanoparticles with Surface Plasmon Resonance Imaging Measurements. ACS Nano 2017, 11, 7447-7456. 
45. Jiang, D.; Jiang, Y. Y.; Li, Z. M.; Liu, T.; Wo, X.; Fang, Y. M.; Tao, N. J.; Wang, W.; Chen, H. Y. Optical Imaging of Phase Transition and Li-Ion Diffusion Kinetics of Single LiCoO2 Nanoparticles During Electrochemical Cycling. J. Am. Chem. Soc. 2017, 139, 186-192.

46. Thambi, V.; Kar, A.; Ghosh, P.; Khatua, S. Light-Controlled in Situ Bidirectional Tuning and Monitoring of Gold Nanorod Plasmon via Oxidative Etching with FeCl3. J. Phys. Chem. C 2018, 122, 24885-24890.

47. Carattino, A.; Khatua, S.; Orrit, M. In situ tuning of gold nanorod plasmon through oxidative cyanide etching. Phys. Chem. Chem. Phys. 2016, 18, 15619-15624.

48. Al-Zubeidi, A.; Hoener, B. S.; Collins, S. S. E.; Wang, W.; Kirchner, S. R.; Hosseini Jebeli, S. A.; Joplin, A.; Chang, W.-S.; Link, S.; Landes, C. F. Hot Holes Assist Plasmonic Nanoelectrode Dissolution. Nano. Lett. 2019, 19, 1301-1306.

49. Cheng, J.; Liu, Y.; Cheng, X. D.; He, Y.; Yeung, E. S. Real Time Observation of Chemical Reactions of Individual Metal Nanoparticles with High-Throughput Single Molecule Spectral Microscopy. Anal. Chem. 2010, 82, 8744-8749.

50. Sun, S. S.; Gao, M. X.; Lei, G.; Zou, H. Y.; Ma, J.; Huang, C. Z. Visually monitoring the etching process of gold nanoparticles by KI/I-2 at single-nanoparticle level using scattered-light dark-field microscopic imaging. Nano Research 2016, 9, 1125-1134.

51. Wang, J.; Zhang, H. Z.; Liu, J. J.; Yuan, D.; Li, R. S.; Huang, C. Z. Time-resolved visual detection of heparin by accelerated etching of gold nanorods. Analyst 2018, 143, 824-828.

52. Zhang, H. Z.; Li, R. S.; Gao, P. F.; Wang, N.; Lei, G.; Huang, C. Z.; Wang, J. Real-time dark-field light scattering imaging to monitor the coupling reaction with gold nanorods as an optical probe. Nanoscale 2017, 9, 3568-3575.

53. Xie, T.; Jing, C.; Ma, W.; Ding, Z. F.; Gross, A. J.; Long, Y. T. Real-time monitoring for the morphological variations of single gold nanorods. Nanoscale 2015, 7, 511-517.

54. Ye, X. C.; Jones, M. R.; Frechette, L. B.; Chen, Q.; Powers, A. S.; Ercius, P.; Dunn, G.; Rotskoff, G. M.; Nguyen, S. C.; Adiga, V. P.; Zettl, A.; Rabani, E.; Geissler, P. L.; Alivisatos, A. P. Single-particle mapping of nonequilibrium nanocrystal transformations. Science 2016, 354, 874-877.

55. Sun, Y. Z.; Fan, X. D. Optical ring resonators for biochemical and chemical sensing. Anal. Bioannal. Chem. 2011, 399, 205-211.

56. Lu, T.; Lee, H.; Chen, T.; Herchak, S.; Kim, J. H.; Fraser, S. E.; Flagan, R. C.; Vahala, K. High sensitivity nanoparticle detection using optical microcavities. Proc. Natl. Acad. Sci. U.S.A. 2011, 108, 59765979.

57. Ward, J. M.; Yang, Y.; Lei, F. C.; Yu, X. C.; Xiao, Y. F.; Nic Chormaic, S. Nanoparticle sensing beyond evanescent field interaction with a quasi-droplet microcavity. Optica 2018, 5, 674-677.

58. Ward, J. M.; Yang, Y.; Lei, F. C.; Yu, X. C.; Xiao, Y. F.; Nic Chormaic, S. Nanoparticle sensing beyond evanescent field interaction with a quasi-droplet microcavity. Optica 2018, 5, 674-677.

59. Barucci, A.; Berneschi, S.; Giannetti, A.; Baldini, F.; Cosci, A.; Pelli, S.; Farnesi, D.; Righini, G. C.; Soria, S.; Conti, G. N. Optical Microbubble Resonators with High Refractive Index Inner Coating for Bio-Sensing Applications: An Analytical Approach. Sensors 2016, 16.

60. Giorgini, A.; Avino, S.; Malara, P.; De Natale, P.; Gagliardi, G. Liquid Droplet Microresonators. Sensors 2019, 19.

61. Madani, A.; Harazim, S. M.; Quinones, V. A. B.; Kleinert, M.; Finn, A.; Naz, E. S. G.; Ma, L. B.; Schmidt, O. G. Optical microtube cavities monolithically integrated on photonic chips for optofluidic sensing. Opt. Lett. 2017, 42, 486-489.

62. Han, K. W.; Kim, J.; Bahl, G. High-throughput sensing of freely flowing particles with optomechanofluidics. Optica 2016, 3, 585-591.

63. Stoian, R.-I.; Bui, K. V.; Rosenberger, A. Silica hollow bottle resonators for use as whispering gallery mode based chemical sensors. J. Opt. 2015, 17, 125011.

64. Ward, J. M.; Dhasmana, N.; Nic Chormaic, S. Hollow core, whispering gallery resonator sensors. Eur. Phys. J.-Special Topics 2014, 223, 1917-1935. 
65. Kippenberg, T. J.; Spillane, S. M.; Vahala, K. J. Demonstration of ultra-high-Q small mode volume toroid microcavities on a chip. Appl. Phys. Lett. 2004, 85, 6113-6115.

66. Armani, A. M.; Vahala, K. J. Heavy water detection using ultra-high-Q microcavities. Optics Letters 2006, 31, 1896-1898.

67. Armani, D. K.; Kippenberg, T. J.; Spillane, S. M.; Vahala, K. J. Ultra-high-Q toroid microcavity on a chip. Nature 2003, 421, 925-928.

68. Black, E. D. An introduction to Pound-Drever-Hall laser frequency stabilization. Am. J. Phys. 2001, $69,79-87$.

69. Barnes, J. A.; Gagliardi, G.; Loock, H. P. Absolute absorption cross-section measurement of a submonolayer film on a silica microresonator. Optica 2014, 1, 75-83.

70. Carmon, T.; Kippenberg, T. J.; Yang, L.; Rokhsari, H.; Spillane, S.; Vahala, K. J. Feedback control of ultra-high-Q microcavities: application to micro-Raman lasers and microparametric oscillators. Opt. Express 2005, 13, 3558-3566.

71. Murugan, G. S.; Petrovich, M. N.; Jung, Y.; Wilkinson, J. S.; Zervas, M. N. Hollow-bottle optical microresonators. Opt. Express 2011, 19, $20773-20784$.

72. Nasir, M. N. M.; Murugan, G. S.; Zervas, M. N. Spectral cleaning and output modal transformations in whispering-gallery-mode microresonators. Journal of the Optical Society of America B-Optical Physics 2016, 33, 1963-1970.

73. Ding, M.; Murugan, G. S.; Brambilla, G.; Zervas, M. N. Whispering gallery mode selection in optical bottle microresonators. Appl. Phys. Lett. 2012, 100.

74. Schunk, G.; Furst, J. U.; Fortsch, M.; Strekalov, D. V.; Vogl, U.; Sedlmeir, F.; Schwefel, H. G. L.; Leuchs, G.; Marquardt, C. Identifying modes of large whispering-gallery mode resonators from the spectrum and emission pattern. Opt. Express 2014, 22, 30795-30806.

75. Ward, J. M.; Yang, Y.; Nic Chormaic, S. Highly Sensitive Temperature Measurements With Liquid-Core Microbubble Resonators. Ieee Photonics Technology Letters 2013, 25, 2350-2353.

76. Hall, J. M. M.; Francois, A.; Afshar, S.; Riesen, N.; Henderson, M. R.; Reynolds, T.; Monro, T. M. Determining the geometric parameters of microbubble resonators from their spectra. J. Opt. Soc. Am. B 2017, 34, 44-51.

77. Murugan, G. S.; Wilkinson, J. S.; Zervas, M. N. Selective excitation of whispering gallery modes in a novel bottle microresonator. Opt. Express 2009, 17, 11916-11925.

78. Davletshin, Y. R.; Lombardi, A.; Cardinal, M. F.; Juve, V.; Crut, A.; Maioli, P.; Liz-Marzan, L. M.; Vallee, F.; Del Fatti, N.; Kumaradas, J. C. A Quantitative Study of the Environmental Effects on the Optical Response of Gold Nanorods. ACS Nano 2012, 6, 8183-8193.

79. Ni, W. H.; Chen, H. J.; Kou, X. S.; Yeung, M. H.; Wang, J. F. Optical fiber-excited surface plasmon resonance spectroscopy of single and ensemble gold nanorods. J. Phys. Chem. C 2008, 112, 8105-8109.

80. Park, K.; Biswas, S.; Kanel, S.; Nepal, D.; Vaia, R. A. Engineering the Optical Properties of Gold Nanorods: Independent Tuning of Surface Plasmon Energy, Extinction Coefficient, and Scattering Cross Section. J. Phys. Chem. C 2014, 118, 5918-5926.

81. Jana, N. R.; Gearheart, L.; Obare, S. O.; Murphy, C. J. Anisotropic chemical reactivity of gold spheroids and nanorods. Langmuir 2002, 18, 922-927.

82. Zou, R. X.; Guo, X.; Yang, J.; Li, D. D.; Peng, F.; Zhang, L.; Wang, H. J.; Yu, H. Selective etching of gold nanorods by ferric chloride at room temperature. CrystEngComm 2009, 11, 2797-2803.

83. Zhao, J.; Nguyen, S. C.; Ye, R.; Ye, B. H.; Weller, H.; Somorjai, G. A.; Alivisatos, A. P.; Toste, F. D. A Comparison of Photocatalytic Activities of Gold Nanoparticles Following Plasmonic and Interband Excitation and a Strategy for Harnessing Interband Hot Carriers for Solution Phase Photocatalysis. ACS Cent. Sci. 2017, 3, 482-488.

84. Masitas, R. A.; Zamborini, F. P. Oxidation of Highly Unstable $<4 \mathrm{~nm}$ Diameter Gold Nanoparticles $850 \mathrm{mV}$ Negative of the Bulk Oxidation Potential. J. Am. Chem. Soc. 2012, 134, 5014-5017.

85. Rodriguez-Fernandez, J.; Perez-Juste, J.; Mulvaney, P.; Liz-Marzan, L. M. Spatially-directed oxidation of gold nanoparticles by Au(III)-CTAB complexes. J. Phys. Chem. B 2005, 109, 14257-14261. 
86. Brongersma, M. L.; Halas, N. J.; Nordlander, P. Plasmon-induced hot carrier science and technology. Nature Nanotech. 2015, 10, 25-34.

87. Zhang, Y. C.; He, S.; Guo, W. X.; Hu, Y.; Huang, J. W.; Mulcahy, J. R.; Wei, W. D. SurfacePlasmon-Driven Hot Electron Photochemistry. Chem. Rev. 2018, 118, 2927-2954.

88. Kale, M. J.; Avanesian, T.; Christopher, P. Direct Photocatalysis by Plasmonic Nanostructures. ACS Catalysis 2014, 4, 116-128.

89. Linic, S.; Aslam, U.; Boerigter, C.; Morabito, M. Photochemical transformations on plasmonic metal nanoparticles. Nature Mat. 2015, 14, 567-576.

90. Besteiro, L. V.; Kong, X. T.; Wang, Z. M.; Hartland, G.; Govorov, A. O. Understanding HotElectron Generation and Plasmon Relaxation in Metal Nanocrystals: Quantum and Classical Mechanisms. ACS Photonics 2017, 4, 2759-2781.

91. Hartland, G. V.; Besteiro, L. V.; Johns, P.; Govorov, A. O. What's so Hot about Electrons in Metal Nanoparticles? Acs Energy Letters 2017, 2, 1641-1653.

92. Wu, K.; Chen, J.; McBride, J. R.; Lian, T. Efficient hot-electron transfer by a plasmon-induced interfacial charge-transfer transition. Science 2015, 349, 632-635.

93. Minutella, E.; Schulz, F.; Lange, H. Excitation-Dependence of Plasmon-Induced Hot Electrons in Gold Nanoparticles. J. Phys. Chem. Lett. 2017, 8, 4925-4929.

94. Kamarudheen, R.; Castellanos, G. W.; Kamp, L. P. J.; Clercx, H. J. H.; Baldi, A. Quantifying Photothermal and Hot Charge Carrier Effects in Plasmon-Driven Nanoparticle Syntheses. ACS Nano 2018, $12,8447-8455$.

95. Zhou, L. A.; Swearer, D. F.; Zhang, C.; Robatjazi, H.; Zhao, H. Q.; Henderson, L.; Dong, L. L.; Christopher, P.; Carter, E. A.; Nordlander, P.; Halas, N. J. Quantifying hot carrier and thermal contributions in plasmonic photocatalysis. Science 2018, 362, 69-72.

96. Wu, C. Y.; Wolf, W. J.; Levartovsky, Y.; Bechtel, H. A.; Martin, M. C.; Toste, F. D.; Gross, E. High-spatial-resolution mapping of catalytic reactions on single particles. Nature 2017, 541, $511-515$.

97. Cortes, E.; Xie, W.; Cambiasso, J.; Jermyn, A. S.; Sundararaman, R.; Narang, P.; Schlucker, S.; Maier, S. A. Plasmonic hot electron transport drives nano-localized chemistry. Nat. Commun. $2017,8$.

98. Schlather, A. E.; Manjavacas, A.; Lauchner, A.; Marangoni, V. S.; DeSantis, C. J.; Nordlander, P.; Halas, N. J. Hot Hole Photoelectrochemistry onAu@SiO2@Au Nanoparticles.J. Phys. Chem. Lett. 2017, 8, 2060-2067.

99. Kim, Y.; Torres, D. D.; Jain, P. K. Activation Energies of Plasmonic Catalysts. Nano. Lett. 2016, 16, 3399-3407.

100. Smith, J. G.; Jain, P. K. The Ligand Shell as an Energy Barrier in Surface Reactions on Transition Metal Nanoparticles. J. Am. Chem. Soc. 2016, 138, 6765-6773.

101. Ruijgrok, P. V.; Verhart, N. R.; Zijlstra, P.; Tchebotareva, A. L.; Orrit, M. Brownian Fluctuations and Heating of an Optically Aligned Gold Nanorod. Phys. Rev. Lett. 2011, 107.

102. Trojek, J.; Chvatal, L.; Zemanek, P. Optical alignment and confinement of an ellipsoidal nanorod in optical tweezers: a theoretical study. J. Opt. Soc. Am. A. 2012, 29, 1224-1236.

103. Xu, X. H.; Cheng, C.; Zhang, Y.; Lei, H. X.; Li, B. J. Scattering and Extinction Torques: How Plasmon Resonances Affect the Orientation Behavior of a Nanorod in Linearly Polarized Light. J. Phys. Chem. Lett. 2016, 7, 314-319.

104. Tong, L. M.; Miljkovic, V. D.; Kall, M. Alignment, Rotation, and Spinning of Single Plasmonic Nanoparticles and Nanowires Using Polarization Dependent Optical Forces. Nano. Lett. 2010, 10, 268-273. 105. Yan, Z. J.; Sweet, J.; Jureller, J. E.; Guffey, M. J.; Pelton, M.; Scherer, N. F. Controlling the Position and Orientation of Single Silver Nanowires on a Surface Using Structured Optical Fields. ACS Nano 2012, 6, 8144-8155.

106. Yang, Y.; Saurabh, S.; Ward, J. M.; Nic Chormaic, S. High-Q, ultrathin-walled microbubble resonator for aerostatic pressure sensing. Opt. Express 2016, 24, 294-299.

107. Henze, R.; Seifert, T.; Ward, J.; Benson, O. Tuning whispering gallery modes using internal aerostatic pressure. Opt. Lett. 2011, 36, 4536-4538. 
108. Cosci, A.; Quercioli, F.; Farnesi, D.; Berneschi, S.; Giannetti, A.; Cosi, F.; Barucci, A.; Conti, G. N.; Righini, G.; Pelli, S. Confocal reflectance microscopy for determination of microbubble resonator thickness. Opt. Express 2015, 23, 16693-16701. 
Other files

Microbubble SI ChemRxiv v2.pdf (2.75 MiB)

view on ChemRxiv • download file 